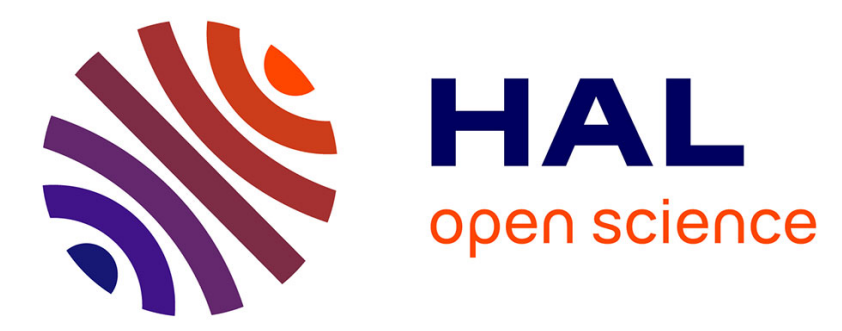

\title{
SIIO et amélioration de la qualité des données: analyse de la synchronisation des données par catalogues électroniques
}

François de Corbière

\section{- To cite this version:}

François de Corbière. SIIO et amélioration de la qualité des données: analyse de la synchronisation des données par catalogues électroniques. Systèmes d'Information et Management, 2011, 16 (3), pp.45-79. 10.3917/sim.113.0045 . hal-00949824

\section{HAL Id: hal-00949824 \\ https://hal.science/hal-00949824}

Submitted on 6 Jul 2021

HAL is a multi-disciplinary open access archive for the deposit and dissemination of scientific research documents, whether they are published or not. The documents may come from teaching and research institutions in France or abroad, or from public or private research centers.
L'archive ouverte pluridisciplinaire HAL, est destinée au dépôt et à la diffusion de documents scientifiques de niveau recherche, publiés ou non, émanant des établissements d'enseignement et de recherche français ou étrangers, des laboratoires publics ou privés. 


\title{
SIIO et amélioration de la qualité des données : analyse de la synchronisation des données par catalogues électroniques.
}

\author{
François de Corbière \\ Ecole des Mines de Nantes \& LEMNA
}

\section{Résumé :}

L'utilisation de systèmes d'information interorganisationnels (SIIO) est généralement présentée comme un moyen pour échanger des données de qualité. Toutefois, il n'existe pas de recherches qui expliquent en profondeur comment l'implémentation de technologies de l'information dans un processus d'échange interorganisationnel peut contribuer à l'amélioration de la qualité des données échangées. En s'appuyant sur la synchronisation des données produit, données à caractère contextuel, par catalogues électroniques entre industriels et distributeurs, cet article présente les caractéristiques des SIIO sources d'amélioration de diverses dimensions de la qualité des données. De plus, les études de cas menées permettent de mettre en exergue diverses structures de SIIO issus de l'interconnexion entre les systèmes d'émission et les systèmes de réception mis en œuvre par les industriels et les distributeurs. La valeur des diverses structures de SIIO, et notamment des SIIO hybrides comparativement aux formes extrêmes, est alors discutée sur la base de leur contribution à l'amélioration de la qualité des données.

Mots clés : qualité des données, systèmes d'information interorganisationnels, formes de SIIO, synchronisation des données, catalogues électroniques.

\begin{abstract}
:
The question of data quality has always been understood to be an important area of research; but after 40 years, there is still a lack of research dealing with the quality of data exchanged between organizations. Even if the literature recognizes that Interorganizational Information Systems (IOSs) use can improve data quality, no research analyzes in depth how data quality can be improved by the implementation of an IOS. In this paper, we focus on electronic catalogues that are implemented to support product data synchronization between consumer goods and retailing industries, and we aim at understanding their contribution to data quality improvement. Emerging from case studies of retailers and manufacturers, our results show that electronic catalogues can contribute to the improvement of several data quality dimensions. Moreover, analysis of interconnections between sending and receiving systems leads to underline diverse structures of IOSs. These diverse forms, and especially hybrid forms compared to extreme forms, allow discussing their value by considering their contribution to data quality improvement.
\end{abstract}

Keywords: Data quality, Interorganizational Information Systems, IOS forms, electronic catalogues, data synchronization. 


\section{Introduction}

Indissociables d'un échange entre organisations, les relations interorganisationnelles voient leur performance intimement liée à la coordination des échanges entre leurs participants (Van de Ven et Walker, 1984). En considérant, dans la lignée de la théorie de la coordination (Malone et Crowston, 1990), que la coordination repose avant tout sur le traitement d'informations, une meilleure gestion des informations constitue une source de création de valeur pour les organisations interreliées (Porter et Millar, 1985). Ce postulat se traduit par le développement et l'utilisation de systèmes d'information interorganisationnels (SIIO) (Barrett et Konsynski, 1982 ; Bensaou, 1997 ; Rai et Tang, 2010).

Parmi les bénéfices généralement associés à la mise en place de SIIO pour les entreprises, certains auteurs mentionnent l'amélioration de la qualité de l'information (Bergeron et Raymond, 1992 ; Legner and Schemm, 2008), notamment quand l'intégration des données est réalisée (Malone et al., 1987 ; Mukhopadhyay et Kekre, 2002). Mais les recherches qui s'intéressent spécifiquement à la question de la qualité des données dans les SIIO restent peu nombreuses malgré les enjeux associés (Nicolaou et McKnight, 2006). Dans une société qui s'informatise de plus en plus, la capacité des technologies de l'information à apporter des informations de qualité aux utilisateurs ou à permettre le transfert de données de qualité devient fondamentale (Zmud, 2005). Dès lors, un axe d'évaluation des SI, et plus particulièrement des SIIO, réside dans leur capacité à permettre l'échange de données de qualité (Madnick et al., 2009). Se pose ainsi la problématique de la capacité des SIIO mis en œuvre par des partenaires commerciaux à supporter l'amélioration de la qualité des données échangées.

La qualité des données est aujourd'hui reconnue comme un concept multi dimensionnel (Wang et Strong, 1996 ; Lee et al., 2002 : Madnick et al., 2009), mais les recherches qui considèrent l'amélioration de la qualité des données dans les SIIO ne questionnent pas empiriquement ces dimensions. D'un point de vue théorique et managérial, une clarification des dimensions de la qualité des données pouvant être améliorées par la mise en place de SIIO présente un double intérêt. D'une part, c'est l'opportunité d'ouvrir la boîte noire de la qualité des données en détaillant les dimensions impactées par l'électronisation des échanges. D'autre part, la mise en exergue de la manière dont les SIIO influencent positivement la qualité des données permet d'enrichir le postulat communément admis que les SIIO contribuent à l'amélioration de la qualité des données (Mukhopadhyay et Kekre, 2002 ; Legner et Schemm, 2008).

Dans ce cadre, cet article vise plus particulièrement à comprendre ce qu'attendent les entreprises au niveau des différentes dimensions de la qualité des données lorsqu'elles mettent en œuvre un SIIO. C'est pourquoi cette recherche se concentre sur des SIIO en cours de construction, liés à la synchronisation des données entre les bases de données produit dans le secteur de la grande distribution (Nakatani et al., 2006 ; Legner et Schemm, 2008 ; Isaac et Volle, 2008 ; de Corbière et Rowe, 2009). Les systèmes de synchronisation de données permettent le partage à tout instant des mêmes données sur les produits pour lesquels les partenaires commerciaux sont en affaire. Mis en œuvre sur la base de la technologie des catalogues électroniques, ces SIIO sont particulièrement intéressants car ils ont notamment pour objectif l'amélioration de la qualité des données échangées entre industriels et distributeurs (Legner et Schemm, 2008, de Corbière et Rowe, 2011). La qualité des données produit se pose en effet comme un véritable défi pour ces entreprises ${ }^{1}$. De plus, l'échange de fiches produit, données caractérisant un produit pour lequel les partenaires sont en affaire et donc données à caractère contextuel (Legner et Schemm, 2008), est un domaine beaucoup moins investi que l'échange de données à caractère transactionnel par Echange de Données

\footnotetext{
${ }^{1}$ http://www.gs1.fr/gs1_fr/travaux_en_cours/le_defi_de_la_qualite_des_donnees
} 
Informatisées (EDI) ou l'échange de données liées à la négociation par les places de marché électronique (Caby-Guillet et al., 2007). Conformément aux conseils de Robey, Im et Wareham (2008), nous délaissons ainsi les SIIO de type EDI qui présentent aujourd'hui certaines limites pour la conceptualisation théorique en nous concentrant sur d'autres SIIO. Pour traiter de la problématique dans ce contexte, la caractérisation des SIIO mis en œuvre pour synchroniser les données produit entre industriels et distributeurs est nécessaire, tout comme l'identification de ce qu'en attendent les entreprises en termes d'amélioration des dimensions de la qualité des données. Les caractéristiques des SIIO et les dimensions de la qualité des données pourront alors être analysées conjointement pour répondre à la question de recherche principale : comment les SIIO mis en œuvre pour synchroniser les données produit entre industriels et distributeurs peuvent contribuer à l'amélioration des dimensions de la qualité des données échangées?

Pour adresser la problématique et plus particulièrement la question de recherche, une revue de littérature est menée sur les concepts qui les sous-tendent. Suite à l'explicitation de la méthodologie, les résultats sont présentés en deux temps : tout d'abord la caractérisation des SIIO qui émergent pour échanger des fiches produit puis les effets attendus de l'électronisation des échanges sur l'amélioration de différentes dimensions de la qualité des données. Une discussion sur les dimensions de la qualité des données présentées comme pouvant être améliorées par la synchronisation des données est enfin menée conjointement à une discussion sur les capacités des différentes formes de SIIO à supporter cette amélioration de la qualité des données.

\section{Cadre théorique de la contribution des SIIO à l'amélioration de la qualité des données}

Dans cette partie, une revue de la littérature est menée sur les deux concepts forts de la problématique, la qualité des données d'une part et les SIIO d'autre part. S'en suit la présentation du cadre d'analyse, construit dans le but de comprendre les effets attendus des SIIO sur l'amélioration de la qualité des données dans le contexte de la synchronisation des données sur les produits.

\subsection{La qualité des données}

Si l'on s'en tient aux définitions accordées aux termes "données" et "informations", une donnée est un signal, alors que l'information est issue de la transformation de cette donnée par un processus cognitif qui lui donne du sens (Marciniak et Rowe, 2009). La synchronisation des données produit reflète des échanges depuis les bases de données des industriels jusqu'aux bases de données des distributeurs, et non à la transformation de ces données en informations par l'organisation réceptrice. Ainsi, nous traitons davantage de la qualité des données que de la qualité de l'information. Toutefois, Khan, Pierce et Melkas (2004) ont démontré qu'il n'existait pas d'éléments permettant de distinguer l'utilisation des termes «qualité des données » et «qualité de l'information » dans la littérature spécialisée sur ce thème. Sont ainsi utilisées dans la revue de la littérature les recherches traitant de la qualité des données et celles traitant de la qualité de l'information.

Trouvant sa source dans la théorie de l'information et dans la multi dimensionnalité du concept de qualité, la décomposition de la qualité des données en plusieurs dimensions n'est pas récente. En effet, de nombreux académiciens se sont attachés à conceptualiser la qualité des données en un certain nombre de dimensions (voir par exemple Ballou et Pazer, 1995 ; Wand et Wang, 1996). On retrouve cette multi dimensionnalité de la qualité des données dans divers champs de recherche, 
comme par exemple pour expliquer la performance en veille stratégique (Lesca et Lesca, 1995), pour évaluer la satisfaction des utilisateurs du SI à la suite des travaux fondateurs de Bailey et Pearson (1983), pour traiter du succès de SI (Delone et McLean, 2003), ou pour discuter de la pertinence du contenu de sites Web (Nicolaou et McKnight, 2006).

Finalement, la plupart de ces recherches proposent a priori des dimensions de la qualité des données et les mesurent pour analyser leur impact sur une variable dépendante. La recherche menée par Wang et Strong (1996) diffère de ces approches. Cette recherche, trop méconnue dans le champ des systèmes d'information, est particulièrement intéressante dans son design. Ces auteurs, comme l'indique le titre de leur article, cherchent à comprendre ce que signifie la qualité des données pour les utilisateurs et non à la mesurer. Depuis une liste de près 179 attributs alloués à la qualité des données dans la littérature, ils réalisent une recherche en plusieurs étapes, par une analyse thématique à partir d'entretiens et par une analyse factorielle à partir de 350 questionnaires, pour faire émerger la décomposition suivante en 4 catégories et 15 dimensions (figure 1). Le regroupement des dimensions dans les quatre catégories précitées est souvent discuté (Lee et al., 2002), mais le spectre couvert par les 15 dimensions englobe les caractéristiques attribuées à la qualité des données dans l'ensemble des recherches traitant de ce thème. La définition accordée à chacune de ces dimensions est proposée en annexe 1.

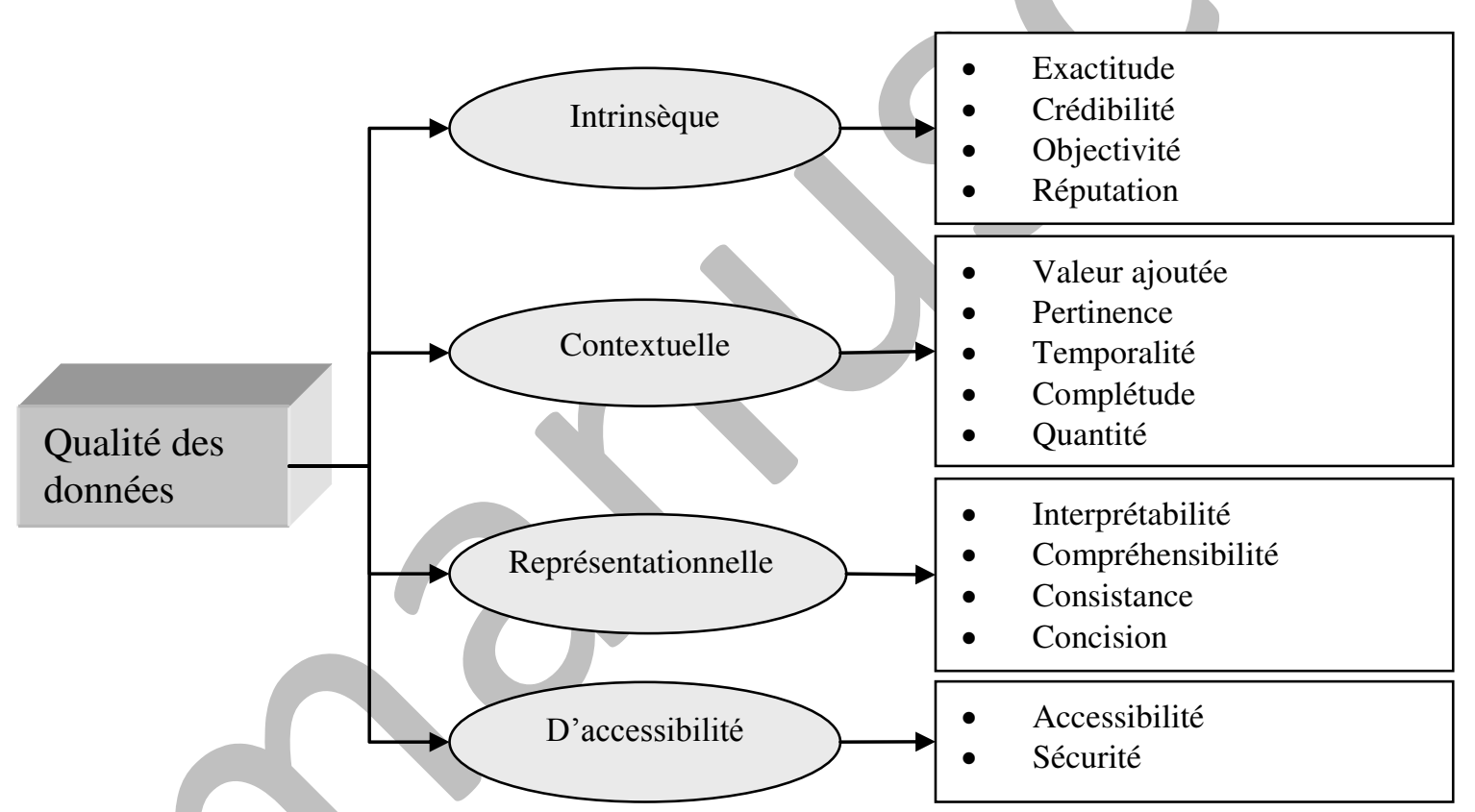

Figure 1: les dimensions de la qualité des données (Wang and Strong, 1996)

Dans l'article introductif (Madnick et al., 2009) du Journal of Data and Information Quality lancé en 2009, les auteurs présentent Wang et Strong (1996) comme la référence fondamentale pour les chercheurs et praticiens qui travaillent sur la définition de la qualité des données. Poursuivant notamment l'objectif d'identifier les dimensions de la qualité des données pour lesquelles une amélioration est attendue par la mise en œuvre de SIIO, nous mobilisons ainsi les 15 dimensions de Wang et Strong (1996) pour analyser l'apport que peut avoir le développement de l'électronisation des échanges sur chacune d'entre elles. Cela permettra ainsi d'enrichir la littérature existante sur les SIIO qui tient compte de la qualité des données ou de l'information. En effet, ces recherches posent un certain nombre de dimensions de la qualité des données a priori pour tester la performance du SIIO ou l'intention d'adopter un SIIO. Par exemple, Baile et Hassairi (2001) mesurent la qualité de 
l'information perçue dans l'entreprise lors de la mise en œuvre de l'EDI à travers la disponibilité, l'exactitude, la complétude, un meilleur accès, une plus grande précision et plus de rapidité. Plus récemment, Hartono, Li, Na et Simpson (2010) ou Li et Lin (2006) mesurent l'amélioration de la qualité de l'information partagée dans les chaînes d'approvisionnement à travers l'utilité, l'exactitude, la temporalité, la complétude ou la fiabilité. Dans la lignée de Iacovou, Benbasat et Dexter (1995), des recherches ont aussi intégré la qualité de l'information en tant que bénéfice perçu dans la décision d'adopter un SIIO : temporalité, exactitude et accessibilité sont alors mesurées (Chewlos et al., 2001). D'autres recherches qui s'intéressent spécifiquement à la qualité des données dans les SIIO ont pour objectif de proposer des méthodes pour améliorer la qualité des données dans un SIIO existant (Scannapieco et al., 2004). Finalement aucune d'entre elles ne propose d'analyser les dimensions pour lesquelles une amélioration peut découler de la mise en place de SIIO. La partie suivante revient sur les concepts qui sous-tendent les SIIO.

\subsection{Les SIIO}

\subsubsection{Les fondements des SIIO}

Défini il y a près de 30 ans dans un article fondateur comme «un système d'information automatisé partagé par au moins deux organisations » (Barrett et Konsynski, 1982, p.94), le SIIO a longtemps été cantonné aux principes de l'échange de données informatisées (EDI), transfert automatisé de données structurées entre les applications de deux entreprises. Un des points clés du SIIO est en effet d'automatiser les flux de données et l'EDI s'est présenté comme un moteur dans la diffusion de tels SIIO. La recherche académique s'est emparée du sujet et la littérature sur ce thème est aujourd'hui abondante (Elgarah et al., 2005 ; Robey et al., 2008). Internet, medium offrant de nombreuses possibilités aux échanges interentreprises, se présente comme une nouvelle opportunité pour développer des SIIO permettant l'automatisation des flux de données d'ordinateur à ordinateur en se fondant sur des standards plus ouverts (Christiaanse et al., 2004 ; Zhu et al., 2006). Car si l'automatisation se présente comme une caractéristique fondamentale des SIIO, elle ne peut se faire sans standardisation (Markus et al., 2006).

Les standards associés aux systèmes d'information interorganisationnels (SIIO) peuvent être définis comme un ensemble de spécifications techniques décrivant les formats de données et les protocoles de communication qui permettent la communication d'ordinateur à ordinateur (Zhu et al., 2006), ceci entre des organisations juridiquement distinctes. Ainsi la migration vers une automatisation des échanges nécessite d'assurer le développement de standards entre les parties prenantes de l'échange, standards qui peuvent prendre deux formes majeures: d'un côté, le standard propriétaire, où une entreprise impose son standard à ses partenaires et d'un autre côté, le standard sectoriel partagé par toutes les entreprises du secteur. Et, comme l'ont souligné Christiaanse, Van Diepen et Damsgaard (2004), "les SIIO décrits par Barrett et Konsynski (1982) étaient basés sur des systèmes propriétaires, qui requièrent des investissements conséquents en spécificité des actifs » (p.161). Le développement de systèmes basés sur des standards plus ouverts remet en cause cette conception trop restrictive de la notion de SIIO et ouvre la voie à d'autres formes de SIIO que nous retrouvons dans diverses typologies (Robey et al., 2008).

\subsubsection{Typologies de SIIO}

Il existe dans la littérature plusieurs typologies de SIIO, qui se fondent sur des bases théoriques différentes, mais qui relèvent toutes de la notion d'interconnexion électronique (Elgarah et al., 
2005). En effet, la mise en place d'un SIIO consiste à interconnecter électroniquement les organisations participantes, pour échanger des données, notamment sur les produits et sur les prix (Bernasconi, 1996). Parmi les différentes typologies de SIIO, notre problématique incite à se concentrer sur celles qui tiennent compte de la coordination des échanges. Dans cette perspective, deux grandes familles de typologies se dégagent de la littérature (Rodon et Sesé, 2010), celles issues de l'analyse des SIIO par l'interdépendance et celles qui ont adapté aux échanges électroniques les structures économiques fondamentales que sont les hiérarchies et les marchés.

La coordination par l'interdépendance est un thème de recherche classique qui se présente comme un aspect fondamental des relations interorganisationnelles (Van de Ven et Walker, 1984). Un courant de littérature s'est appliqué à typer les SIIO en fonction de l'interdépendance qui relie les entreprises. Kumar et van Dissel (1996) ont identifié trois types de SIIO, présentées dans la figure 2. Parmi les différents niveaux d'interdépendances (technique, informationelle, organisationnelle), nous nous concentrons dans cet article sur l'interdépendance technique (Clergeau et Rowe, 2005), qui renvoie aux modalités de partage de technologies pour supporter un échange automatisé de données.

SIIO type chaîne de valeur, représentatif de l'interdépendance séquentielle

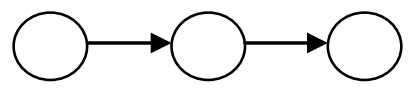

SIIO en réseau, représentatif de l'interdépendance réciproque

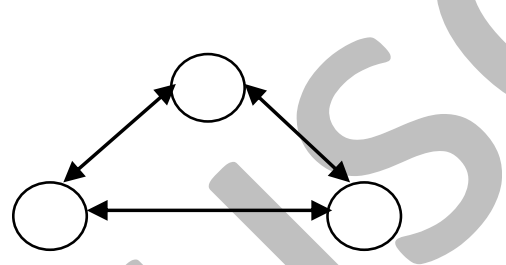

SIIO de ressources mises en commun, représentatif de l'interdépendance en pool

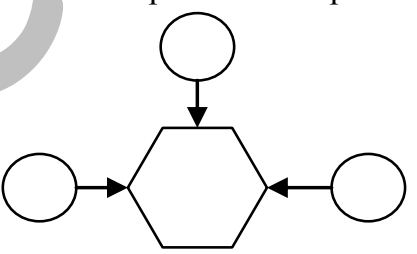

Figure 2 : types de SIIO dans la perspective de l'interdépendance (Kumar et van Dissel, 1996)

Malone, Yates et Benjamin (1987) ont apporté une contribution fondamentale sur le rôle de l'électronisation des échanges entre entreprises. Leur article propose d'adapter aux échanges de données les deux mécanismes fondamentaux de l'économie pour coordonner les transactions de biens et de services : le marché et la hiérarchie (Williamson, 1985). L'électronisation des échanges de données se présente comme pouvant être réalisée à travers des échanges «many-to-many » grâce à des marchés électroniques permettant de comparer de façon plus transparente les produits ou les services, ou à travers des échanges «one-to-one » grâce à des hiérarchies électroniques permettant de relier directement un acheteur avec un vendeur prédéterminé. Dans ce courant, c'est l'article de Choudhury (1997) qui retient le plus notre attention, car il présente une typologie en fonction des logiques qui guident les liaisons électroniques plus que des logiques marchandes : les dyades électroniques représentent la multiplication de SIIO bilatéraux dans lesquels chaque entreprise établit une logique de liens individuels avec ses partenaires (figure 3); les SIIO multilatéraux permettent à une entreprise de communiquer avec un potentiel illimité de partenaires, en passant par une logique de lien unique vers l'extérieur (figure 3). Les SIIO issus d'interconnexions entre entreprises sous la forme de dyades électroniques ont longuement été étudiés à travers l'EDI (Baile et Hassairi, 2001 ; Choudhury, 1997 ; Christiaanse et al., 2004 ; Rodon et Sesé, 2010). Les SIIO multilatéraux se développent depuis quelques années, notamment sous la forme de places de marché électronique, où un intermédiaire électronique centralise les communications (Christiaanse et al., 2004 ; Soh et al., 2006 ; Dominguez, 2009 ; Rodon et Sesé, 2010). 

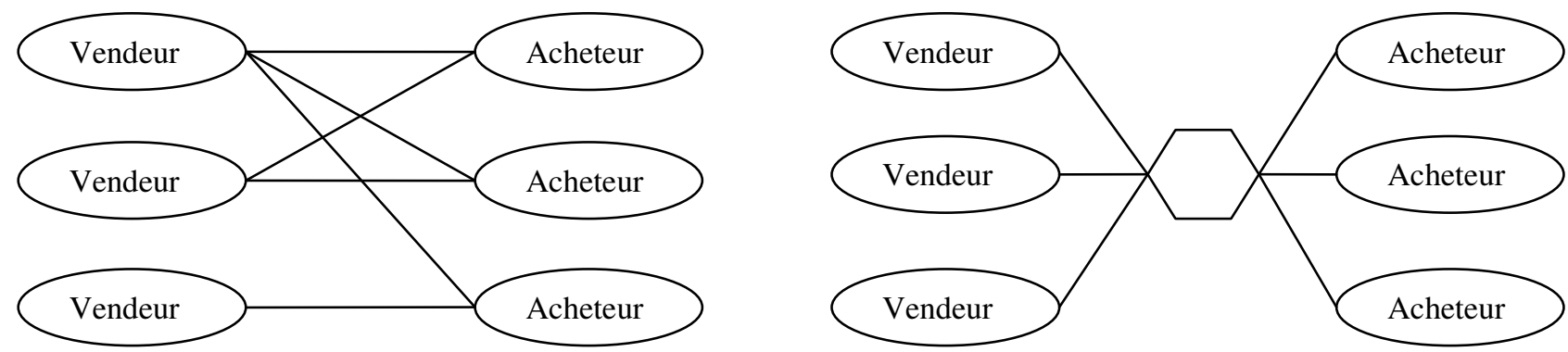

Figure 3 : Les dyades électroniques et le SIIO multilatéral de Choudhury (1997)

En occultant l'aspect marchand de la transaction, puisque pour notre recherche, l'échange de données à caractère contextuel n'a pas pour but de réguler la transaction des produits entre les partenaires commerciaux, ces deux structures de SIIO peuvent émerger. Nous remarquons que les dyades électroniques reflètent une interdépendance technique séquentielle entre clients et fournisseurs, tandis que le SIIO multilatéral propose un centre névralgique qui fédère et distribue les données, dans une interdépendance technique en pool. Au niveau de l'interdépendance technique (Clergeau et Rowe, 2005), nous retrouvons donc des similitudes dans les typologies de Kumar et van Dissel (1996) et de Choudhury (1997).

\subsection{SIIO de synchronisation des données et cadre d'analyse}

\subsubsection{La synchronisation des données produit}

Pour gérer son catalogue produit interne, ou référentiel, un distributeur a besoin de recevoir de la part de l'industriel la fiche produit. La fiche produit est définie comme un ensemble de données caractérisant le produit : données d'identification, données commerciales, données logistiques et données marketing. Ces données servent à la constitution ou à la mise à jour des référentiels des distributeurs, à partir desquels les données sont accessibles pour les processus avals liés aux approvisionnements. Dans le cas des fiches produit, nous avons donc affaire à un transfert de données depuis le système d'information interne de l'industriel jusqu'à celui du distributeur. Suite à l'échec relatif de l'EDI pour échanger ce type de messages au cours du siècle passé (Guilloux, 1995), notamment à cause du caractère peu structuré du modèle de données de la fiche produit, l'idée de la transmission électronique des fiches produit a été relancée depuis le début des années 2000 par la Global Data Synchronization (GDS). La synchronisation des données repose sur l'électronisation des échanges de fiches produit par l'utilisation de catalogues électroniques, définis comme des bases de données produit accessibles par Internet.

L'idée majeure consiste à mettre en place un réseau mondial de catalogues électroniques (Nakatani et al., 2006 ; Isaac et Volle, 2008 ; Legner and Schemm, 2008), appelé GDSN (Global Data Synchronization Network). Son slogan, «publier une fois, diffuser à tous, souscrire une fois, recevoir de tous » (GS1, 2006) renvoie l'idée que chaque entreprise monte une seule connexion avec l'extérieur pour communiquer avec tous ses partenaires. Par rapport aux méthodes traditionnelles faites d'e-mails contenant une feuille Excel attachée, la GDS propose l'échange électronique de fiches produit par des communications multilatérales automatisées et non plus bilatérales avec ressaisie manuelle pour chacun des partenaires commerciaux. Ainsi chacune des entreprises participantes utilise un catalogue électronique qui appartient au réseau GDSN pour que celui-ci gère les communications électroniques de fiches produit par XML (eXtensible Markup Language). 
Toutefois, cette approche ne fait pas l'unanimité, car des technologies ont émergé en parallèle pour échanger électroniquement les fiches produit. C'est notamment le cas du PIM (Product Information Management), dont la fonctionnalité principale est la gestion interne des fiches produit pour les structurer en messages à la frontière du SI de l'entreprise (e-business, 2005). Cet outil est une forme de catalogue électronique interne, qui permet aussi d'envoyer ou de recevoir directement les fiches produit sans faire appel à un intermédiaire externe (Nakatani et al., 2006). De même, certaines entreprises peuvent faire le choix d'utiliser un catalogue électronique externe pour électroniser leurs échanges de fiches produit, mais sans que ce catalogue appartienne au réseau GDSN. Quelque soit leur forme, interne, externe hors GDSN ou externe du GDSN, les catalogues électroniques ont tous pour vocation de développer la synchronisation des données, pour qu'à tout moment, les partenaires commerciaux partagent les mêmes données sur les produits dans leurs propres référentiels.

\subsubsection{Cadre d'analyse de la contribution des SIIO à la qualité des données}

Dans la lignée de Lyytinen et Damsgaard (2011), nous réalisons une analyse configurationnelle des SIIO mis en œuvre pour réaliser la synchronisation des données produit. Ce type d'analyse permet de ne pas penser le SIIO comme un tout, mais de procéder en plusieurs étapes, avec tout d'abord une analyse du système d'information que chaque entreprise met en œuvre pour réaliser ses communications, puis une analyse du SIIO résultant des liaisons électroniques entre les systèmes d'un ensemble d'acteurs. Nous proposons ainsi de considérer le SIIO comme le résultat de l'interconnexion entre les systèmes d'émission et de réception mis en place par un ensemble de partenaires (de Corbière et Rowe, 2009). Il est particulièrement pertinent d'orienter l'analyse de cette manière car les technologies de synchronisation de données sont des technologies basées sur les standards ouverts d'Internet (Legner et Schemm, 2008 ; Isaac et Volle, 2008). C'est en ce sens qu'elles sont différentes des technologies EDI (Zhu et al., 2006), notamment car elles autorisent la réduction conséquente de l'ajustement mutuel entre entreprises pour réaliser l'interconnexion (Christiansee et al., 2004). De ce fait, la plus grande interconnectivité entre les technologies des différents partenaires les laisse davantage libres dans l'implémentation de la partie du SIIO dont ils sont respectivement responsables.

Pour réaliser la synchronisation des données avec ses partenaires, chaque entreprise met en place un catalogue électronique (Legner et Schemm, 2008), qui combine les fonctionnalités d'une base de données pour stocker et organiser les données à échanger et les fonctionnalités permettant leur échange : la structuration des données dans des messages et l'interconnectivité de son catalogue, avec la standardisation des données et des protocoles de communication (Zhu et al., 2006). C'est enfin l'interconnexion effective entre les catalogues électroniques des partenaires qui permet de réaliser la synchronisation des données entre leurs bases de données internes respectives par l'automatisation des flux de données à trois étapes du processus d'échange : lors de l'émission des données depuis la base de données interne de l'émetteur jusqu'à son catalogue électronique (interne ou externe) ; lors de la transmission des données consistant à réaliser l'échange des données entre le catalogue électronique de l'émetteur et celui du récepteur ; lors de la réception des données depuis le catalogue électronique (interne ou externe) du récepteur jusqu'à sa base de données interne.

En synthèse, pour caractériser les systèmes d'émission et de réception, nous tenons compte de la structure du système (Choudhury, 1997 ; Kumar et van Dissel, 1996) mis en place par chaque partenaire (lien multilatéral ou liens dyadiques), de l'automatisation (Elgarah et al., 2005) et de la standardisation (Markus et al., 2006; Zhu et al., 2006). Comme le présente la figure 5, nous proposons de manière générique que le SIIO de synchronisation de données, résultant de 
l'interconnexion entre le système d'émission et le système de réception, permet d'améliorer chacune des 15 dimensions de la qualité des données définies par Wang et Strong (1996). L'objectif principal de la partie empirique est d'affiner cette proposition générique par, d'une part, l'identification des caractéristiques de ce système et, d'autre part, la compréhension de leur contribution à l'amélioration des différentes dimensions de la qualité des données.

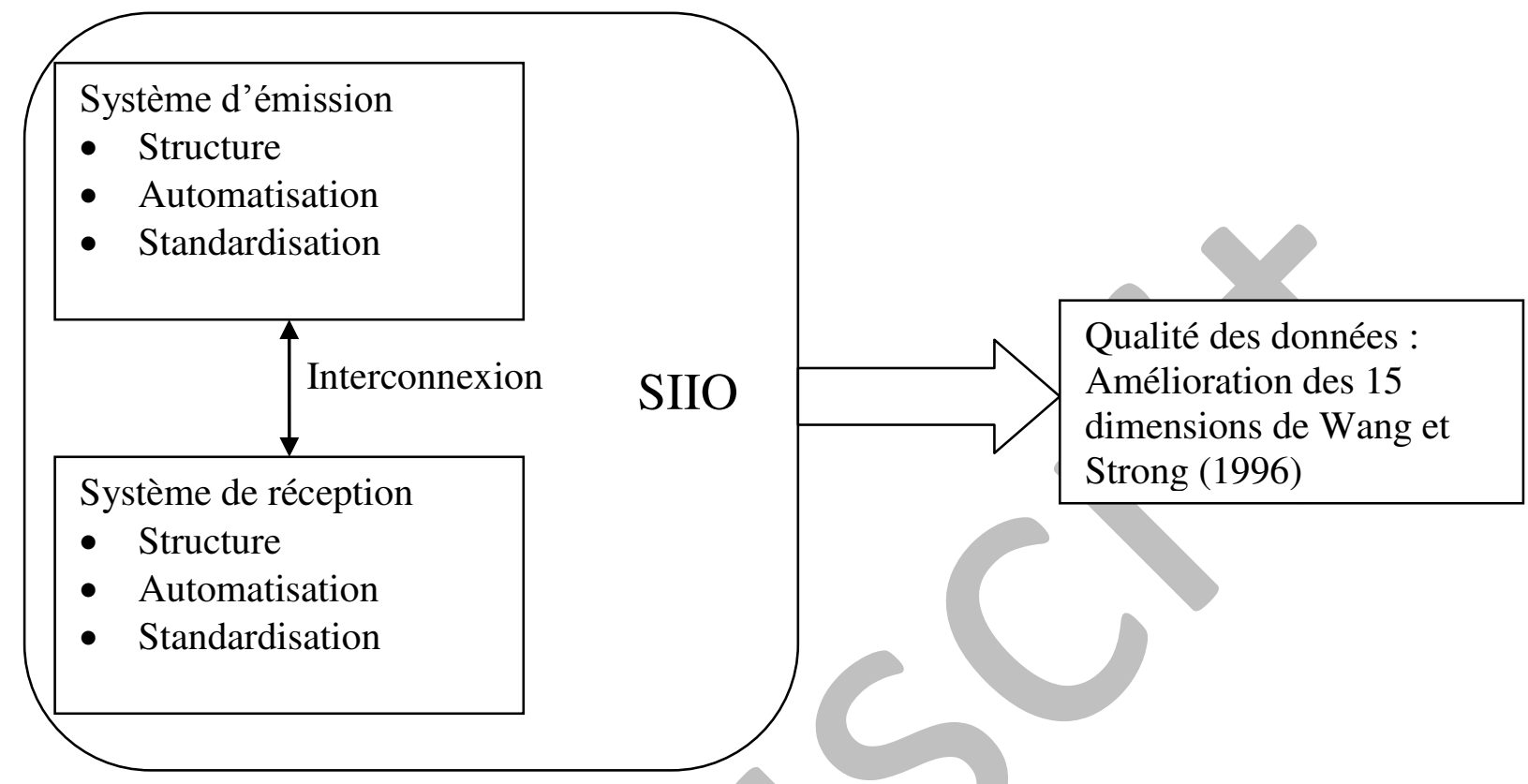

Figure 5 : visualisation de la question de recherche

\section{Le design de la recherche}

Dans cette partie est exposée la méthodologie mise en œuvre pour, d'une part, caractériser les SIIO de synchronisation des données produit et, d'autre part, analyser leurs capacités à soutenir l'amélioration de la qualité des données.

\subsection{Une recherche fondée sur des études de cas multiples}

Devant la posture compréhensive de l'analyse de la capacité des SIIO à soutenir l'amélioration de la qualité des données, une démarche qualitative fondée sur des études de cas a été menée, méthode recommandée pour investir des phénomènes contemporains en émergence dans leur contexte (Hlady-Rispal, 2002 ; Yin, 2003). Conformément à la question de recherche, l'accent est mis, d'une part, sur les choix technologiques des entreprises du secteur de la grande distribution pour échanger des fiches produits par catalogues électroniques et, d'autre part, sur leurs attentes en termes d'amélioration de la qualité des données. L'intérêt des études de cas vis-à-vis d'une démarche quantitative par questionnaire repose notamment sur le fait d'obtenir de la part des acteurs tant une description fine de leur système d'information qu'une explicitation verbale de la valeur du SIIO concernant la qualité des données échangées. Quand on s'intéresse aux relations interorganisationnelles, trois niveaux d'analyse peuvent être considérés (Oliver, 1990) : la dyade, le réseau ou l'entreprise quand on se focalise sur ses relations avec ses partenaires. Devant ces possibilités, nous avons décidé de centrer les cas sur les entreprises, pour comprendre ce qu'elles attendent de la synchronisation des données avec leurs partenaires en termes d'amélioration de la qualité des données. Puis, conformément à l'analyse configurationnelle retenue (Lyytinen et Damsgaard, 2011), le niveau d'analyse est élevé à des réseaux d'entreprises qui structurent des 
configurations particulières de SIIO pour identifier leurs capacités à soutenir l'amélioration de certaines dimensions de la qualité des données.

Pour obtenir une représentation la plus juste possible des formes de SIIO mis en œuvre pour émettre et recevoir les fiches produit par catalogues électroniques dans le secteur, un design d'études de cas multiples (Yin, 2003) a été adopté. C'est en effet l'analyse d'un nombre conséquent d'industriels et de distributeurs qui permet, d'une part d'appréhender la diversité des formes de SIIO résultant de l'interconnexion entre les systèmes d'émission et les systèmes de réception des données, et d'autre part d'obtenir une vision agrégée de la capacité des SIIO à soutenir l'amélioration de la qualité des données. Du côté des distributeurs, leur faible nombre a conduit à ne pas faire d'échantillonnage en intégrant à l'analyse l'ensemble des sept centrales d'achat nationales (Carrefour, Galec (Leclerc), Auchan, Casino, Intermarché, Système U et Provera). Du côté des industriels, un échantillonnage était indispensable. La décision de se concentrer sur les grandes entreprises est issue du fait que, dans l'implémentation de SIIO utilisant de nouvelles technologies, les PME agissent généralement en suiveurs plutôt qu'en prescripteurs (Subramani, 2004). Conformément aux critères de distinction proposés par Lepers (2003), deux types d'industriels forment l'échantillon : dix multinationales telles que Unilever, Nestlé, l'Oréal, Danone ou Coca-Cola et huit nationales telles que Tipiak, Charal, Lavazza, Fleury Michon.

\subsection{Collecte et analyse des données}

La collecte des données s'est déroulée entre 2005 et 2007 dans les 25 entreprises de notre échantillon. Conformément aux prescriptions de Yin (2003), différentes sources de données permettent une triangulation des données, préconisée dans l'étude de cas pour accroître la validité des résultats (Hlady-Rispal, 2002). Les 40 entretiens semi-directifs, d'une moyenne de deux heures environ, ont été réalisés avec des personnes ayant sous leur responsabilité tout ou partie du projet d'implémentation de catalogues électroniques, en se fondant sur le guide d'entretien présenté en annexe 2. Une description plus fine des entretiens est proposée en annexe 3. Cette source de données primaires a été complétée par des sources de données secondaires, et notamment des documents, internes ou externes aux entreprises, relatifs aux projets de synchronisation des données produit. Ces documents ont été utilisés pour comprendre davantage le fonctionnement des échanges de données par catalogue électronique et confirmer les discours des acteurs. Autre source de données secondaires, quatre entretiens ont été réalisés dans des entreprises gérant un catalogue électronique et chez GS1, organisme garant de la standardisation des échanges dans le secteur.

Une analyse thématique, au sens de Miles et Huberman (2003) a alors été menée à partir des données collectées en les codant à l'aide du logiciel QSR N'Vivo 7. Le codage descriptif a consisté à extraire des entretiens, enregistrés et retranscrits intégralement, les items présentant, pour chaque entreprise : l'implémentation de catalogues électroniques, les données intégrées dans les fiches produit, les caractéristiques des flux de données entre les différents outils, les modalités d'interconnexion envisagées avec les choix des partenaires et les attentes en termes d'amélioration de la qualité des données, sur chacune des 15 dimensions de Wang et Strong (1996). C'est par le codage thématique qu'ont été associés les caractéristiques des SIIO de synchronisation des données produit et les effets attendus sur l'amélioration des différentes dimensions de la qualité des données.

L'analyse intra-cas présente les caractéristiques des différents systèmes d'émission et de réception qui émergent dans le secteur pour échanger les fiches produit par l'implémentation de catalogues électroniques. Elle permet aussi d'extraire des indications sur les dimensions de la qualité des 
données qui pourraient être améliorées selon chaque entreprise. Toutefois, ces cas ne permettent pas de répondre significativement à la question de recherche quant à l'amélioration de la qualité des données, du fait du faible volume de données recueillies par cas et de notre posture pour collecter les items présentant la qualité des données. En effet, nous n'avons pas cherché à influencer les réponses des interviewés sur la qualité des données: ainsi nous n'avons pas testé de manière systématique les 15 dimensions de la qualité des données de Wang et Strong (1996). Conformément aux conseils de Diane Strong, c'est tout l'intérêt des entretiens vis-à-vis d'un questionnaire de pouvoir poser des questions ouvertes sur les dysfonctionnements constatés sur les données de la fiche produit avant l'introduction des catalogues électroniques. C'est alors à partir de ces dysfonctionnements que nous avons demandé à nos interlocuteurs l'apport perçu des nouvelles formes d'organisation des échanges pour éradiquer, ou au moins réduire, ces dysfonctionnements.

L'analyse inter-cas vient alors en complément pour répondre à la problématique. D'une part, elle présente les multiples combinaisons d'interconnexion entre les systèmes d'émission et les systèmes de réception. De ce fait, elle permet de mettre en exergue les caractéristiques communes des différents SIIO issus de l'interconnexion entre les systèmes d'émission et les systèmes de réception. D'autre part, grâce au nombre de cas analysés, nous extrayons par agrégation les dimensions de la qualité des données pour lesquelles les acteurs attendent une amélioration par le développement de la synchronisation des données. C'est ainsi dans cette phase que nous répondons à la question de recherche en analysant comment les SIIO de synchronisation des données peuvent contribuer à l'amélioration de la qualité des données dans ses différentes dimensions.

\section{Résultats}

Dans cette partie, nous présentons successivement les résultats des études de cas qui nous permettent d'agréger les grandes tendances sur les systèmes d'émission et de réception des données. L'analyse inter-cas met ensuite en évidence les interconnexions entre ces systèmes et les effets attendus de l'interconnexion en termes d'amélioration des différentes dimensions de la qualité des données. La dernière section présente ainsi la capacité des SIIO de synchronisation de données à supporter l'amélioration de la qualité des données en spécifiant les caractéristiques de ces SIIO qui influencent selon les acteurs chacune des dimensions de Wang et Strong (1996).

\subsection{Présentation des systèmes d'émission et de réception}

Cette section rend compte des résultats des études de cas vis-à-vis des systèmes d'émission et des systèmes de réception mis en œuvre par les entreprises analysées. Nous avons choisi un mode de présentation selon les éléments qui caractérisent les systèmes plutôt que de nous concentrer de manière séquentielle sur les 25 cas analysés.

\subsubsection{Les structures des systèmes d'émission et de réception des données}

Suite au codage des entretiens réalisés chez les 18 industriels de notre échantillon, il émerge par agrégation trois formes de structures pour émettre les fiches produit par l'implémentation de catalogues électroniques (Tableau 1). Ces configurations sont extraites des choix des industriels concernant l'implémentation de catalogues propriétaires, les PIM (Product Information Management) mis en œuvre à l'intérieur du SI de l'industriel, et/ou de catalogues externes partagés, les SDP (Source Data Pool) mis en œuvre à l'extérieur du SI de l'industriel. La première forme, mobilisée par 7 industriels de notre échantillon, consiste à combiner un PIM et un SDP pour 
envoyer les fiches produit aux distributeurs. Les données sont extraites du SI préexistant vers le PIM, qui structure et envoie les fiches produit au SDP. Ainsi, le PIM a pour principale fonction de gérer les fiches produits, c'est-à-dire de structurer l'ensemble des données qui sont rattachées à un produit particulier. Le SDP a alors pour rôle de diriger les fiches produit vers les systèmes de réception des distributeurs. La seconde forme, mobilisée par 7 industriels, correspond à la seule implémentation d'un PIM. Les données extraites du SI vers le PIM sont alors structurées en fiche produit et directement envoyées aux systèmes de réception des distributeurs depuis le PIM. La troisième forme consiste à utiliser uniquement un SDP. Les données sont donc extraites du SI vers le SDP qui se charge de les transmettre sous forme de fiches produit aux distributeurs.

Tableau 1 : les organisations de l'émission et de la réception électronique

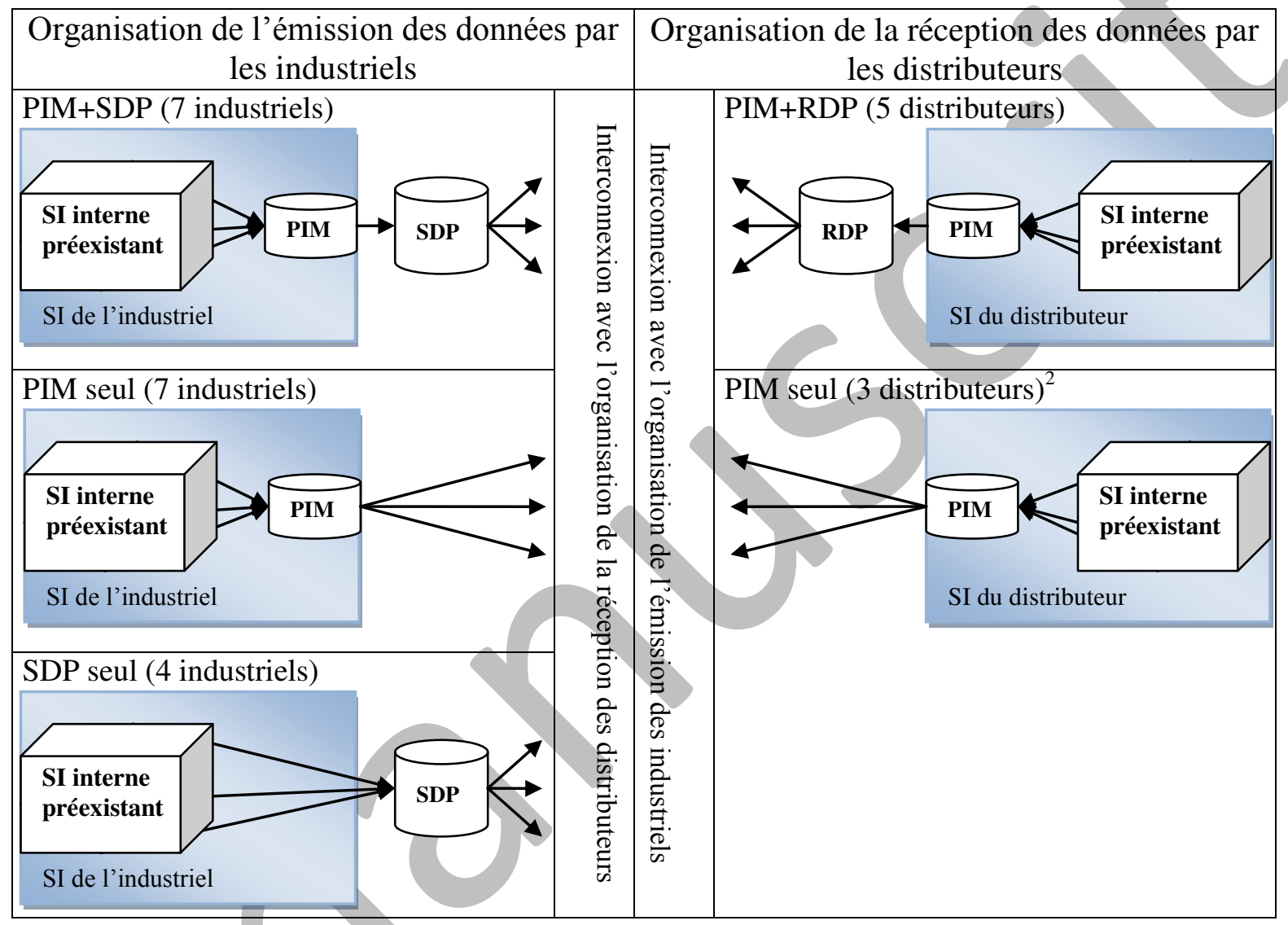

On retrouve du côté des distributeurs plusieurs stratégies d'utilisation de catalogues électroniques pour recevoir les fiches produit. A la différence des industriels, tous les distributeurs mettent en œuvre un PIM, sorte de sas entre les SI externes et leur SI interne pré-existant, qui permet de rediriger les données reçues vers les référentiels. Il en résulte deux modes d'organisation de la réception des fiches produit (Tableau 1). Pour cinq distributeurs, il s'agit de combiner l'implémentation d'un PIM avec un catalogue externe, un RDP (Recipient Data Pool). Le RDP reçoit les fiches produit des systèmes d'émission des industriels et les transfère ensuite au PIM du distributeur. Pour trois distributeurs, puisqu'un d'entre eux a choisi de combiner les deux formes de réception par catalogues électroniques, la stratégie consiste à utiliser leur PIM pour recevoir électroniquement les fiches produit depuis le système d'émission de leurs fournisseurs.

\footnotetext{
${ }^{2}$ Un des sept distributeurs a décidé de coupler les deux types de réception
} 


\subsubsection{De l'automatisation à la standardisation}

Que ce soit pour les systèmes d'émission ou les systèmes de réception, l'automatisation des flux de fiches produit est un des points clés qui justifie l'implémentation de catalogues électroniques, puisque l'automatisation des flux se présente comme une condition pour réaliser la synchronisation des données. En effet, les 7 distributeurs conçoivent leurs systèmes de réception à travers l'automatisation des flux depuis leurs catalogues électroniques externes et internes jusqu'aux référentiels internes, pour y intégrer les données provenant des industriels sans ressaisies manuelles. De même, l'ensemble des industriels de notre échantillon a pour objectif d'automatiser les flux des données entre leurs référentiels, les PIM et les SDP. Sur les 18 industriels, seuls 3 d'entre eux ont décidé de passer par une phase intermédiaire en ressaisissant manuellement les données directement dans leur SDP pour tester la solution avant de paramétrer l'automatisation des flux de données.

Concernant la standardisation, celle des protocoles de communication est une condition sine qua none des projets d'électronisation des échanges de fiches produit. Cette standardisation ne pose fondamentalement pas de problème : l'ensemble des 25 entreprises analysées se tourne vers l'échange de fichiers structurés en XML par Internet, en adéquation avec les préconisations de GS1, l'organisme de standardisation qui fait foi dans le secteur. La connexion entre les catalogues et les référentiels internes se fonde dans certaines entreprises sur des standards propriétaires qui diffèrent du standard défini par GS1, mais les intermédiaires que sont les catalogues électroniques disposent de fonctionnalités permettant le paramétrage de la traduction entre ces standards. Par contre, le standard de contenu, le modèle de données de la fiche produit, est plus discuté. Globalement, à partir du standard EAN.UCC défini par GS1 qui forme de manière unanime la base du modèle de données, trois types de données peuvent être distingués : les données obligatoires, les données complémentaires et les données hors standard. Certaines entreprises (2 distributeurs et 6 industriels) se limitent aux données neutres qui constituent l'ensemble des données obligatoires du standard EAN.UCC défini par GS1. D'autres (3 distributeurs et 7 industriels) demandent, ou acceptent, l'introduction dans les messages des données complémentaires, qui sont intégrées au standard mais qui ne sont pas obligatoires. C'est notamment le cas des données qui permettent la description tarifaire du produit. Les derniers ( 2 distributeurs et 5 industriels) n'avaient pas arrêté leur décision à la fin de l'année 2007. De plus, 2 distributeurs demandent l'introduction de données hors standard, comme par exemple le nom de l'acheteur concerné par la réception de la fiche produit. 8 industriels répondent à leur demande.

Dans la partie suivante, les SIIO résultant de l'interconnexion entre les systèmes d'émission et de réception des fiches produit sont présentés.

\subsection{Caractérisation des SIIO émergents}

Issus de la diversité des systèmes d'émission et de réception, les SIIO résultant de leurs interconnexions présentent de fait des disparités importantes pour l'échange de fiches produit. Pour mettre en exergue les structures des SIIO qui émergent, la figure 5 présente des analyses configurationnelles d'interconnexion entre trois industriels et trois distributeurs parmi six industriels (I1, I2 et I3 utilisant un SDP, et I4, I5 et I6 utilisant seulement un PIM) et six distributeurs (D1, D2 et D3 utilisant un RDP, et D4, D5 et D6 utilisant seulement un PIM). Au regard des systèmes d'émission et des systèmes de réception mobilisant des catalogues électroniques, leur interconnexion met en évidence cinq formes de SIIO : 
- L'interconnexion entre les industriels utilisant un SDP et les distributeurs utilisant un RDP. Dans ce SIIO de type A, chacun des participants met en place une connexion unique vers un catalogue électronique externalisé pour communiquer avec l'ensemble de ses partenaires.

- L'interconnexion entre les industriels et les distributeurs utilisant un PIM sans SDP et RDP. Dans ce SIIO de type B, chaque participant met en place une connexion avec chacun de ses partenaires.

- L'interconnexion entre les industriels utilisant un SDP et les distributeurs n'utilisant pas de RDP. Dans ce SIIO de type C, les industriels utilisent une connexion unique pour communiquer avec tous leurs clients mais les distributeurs sont dans une logique de liens bilatéraux, avec une connexion vers chacun des catalogues externes des industriels.

- L'interconnexion entre les industriels n'utilisant pas de SDP et les distributeurs utilisant un RDP. Dans ce SIIO de type D, les industriels utilisent une connexion avec chacun des catalogues externes des distributeurs qui sont pour leur part dans une logique de lien unique pour communiquer avec tous leurs fournisseurs.

- L'interconnexion où les trois industriels et/ou les trois distributeurs ont des choix disparates, certains utilisant un catalogue externe et d'autres non. Ce SIIO de type E se présente comme une configuration qui combine des logiques de liens bilatéraux et de liens multilatéraux.

Ces formes de SIIO résultant de notre analyse empirique seront mises en perspective dans la discussion en revenant sur la revue de la littérature effectuée dans la première partie.

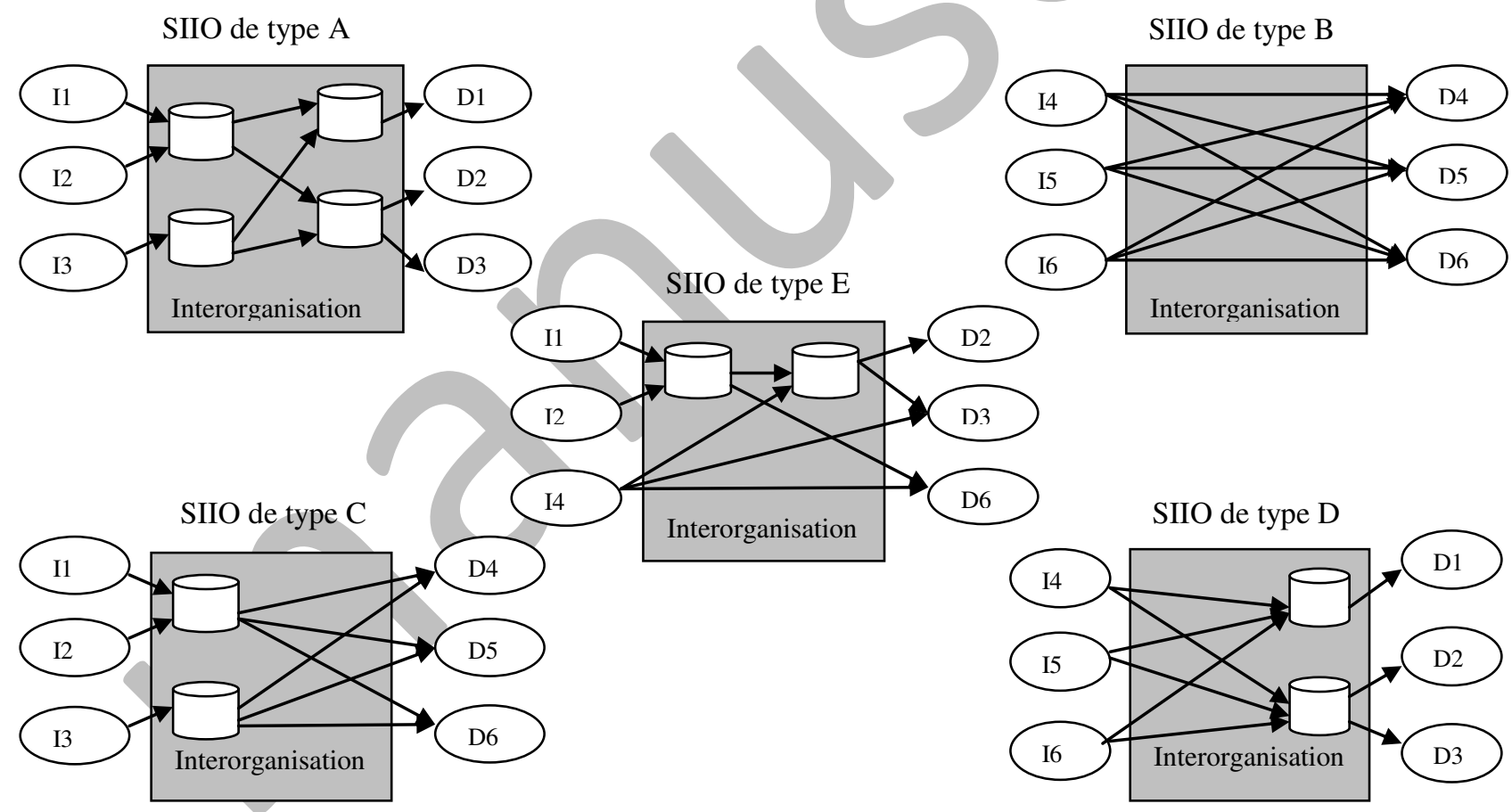

Figure 5 : cinq configurations de SIIO

\subsection{Analyse des effets attendus de l'électronisation des échanges sur l'amélioration de la qualité des données}

Suite à la présentation des formes de SIIO qui émergent dans le secteur, cette partie se concentre sur les effets attendus en termes d'amélioration de la qualité des données. L'électronisation des échanges entre les bases de données internes d'un industriel vers un distributeur peut participer 
selon les acteurs à l'amélioration de plusieurs dimensions de la qualité des données. Le tableau 2 résume les effets attendus de l'implémentation de catalogues électroniques sur l'amélioration des 15 dimensions de Wang et Strong (1996). La deuxième ligne de ce tableau spécifie le nombre d'entreprises qui attendent une amélioration de chacune des 15 dimensions de la qualité des données. L'analyse agrégée des données recueillies dans les 25 entreprises de notre échantillon met en évidence que 12 des 15 dimensions peuvent être améliorées selon les acteurs. Par l'analyse thématique liant les dimensions de la qualité des données et l'électronisation des échanges par catalogues électroniques, cinq éléments des SIIO mis en œuvre sont associés à l'amélioration de certaines de ces dimensions. Les cellules tintées en gris dans le tableau 2 permettent de faire ressortir ce lien. Dans chacune de ces cellules est spécifié le nombre d'entreprises pour lesquelles sont associés l'élément des SIIO mis en œuvre et la dimension sur laquelle une amélioration est attendue.

Tableau 2 : effets attendus de l’implémentation de catalogues électroniques sur la qualité des données.

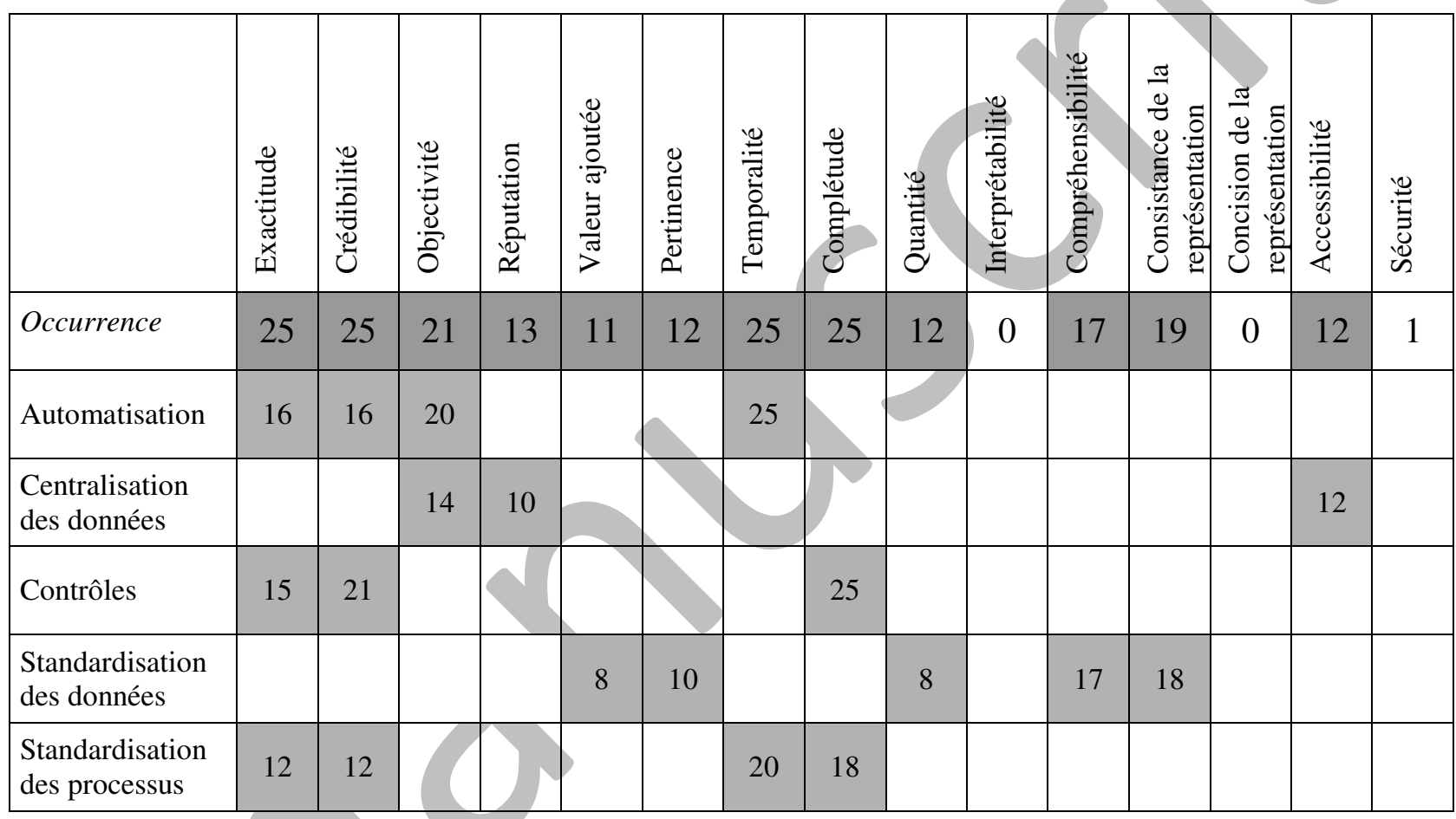

Pour l'automatisation des flux, un certain consensus émerge sur le fait que l'automatisation complète entre les bases de données internes constitue une réelle opportunité pour améliorer l'exactitude, la crédibilité, l'objectivité et la temporalité des données: «La transmission électronique, ça permet d'avoir une information plus juste et plus vite. Ça amplifie les économies » (Dist D). Le risque qu'une donnée soit mal ressaisie disparaît. Ainsi, l'automatisation permet de supprimer le risque de détérioration de l'exactitude ou de la crédibilité des données. En ce sens, comparativement aux échanges antérieurs qui nécessitaient des ressaisies, la fiabilité des données est améliorée : "L'enjeu est de fiabiliser les références des produits. Un problème de non qualité, c'est par exemple une erreur de palettisation, quand on s'en rend compte lors de la livraison en magasin, c'est très gênant. L'utilisation des catalogues électroniques réduit ces risques d'erreurs » (Dist C). De plus, l'automatisation est un gage d'objectivité des données car elle assure que les données ne sont pas modifiées lors de leur transfert: «il y a aussi surtout le fait que, en transmettant de la donnée via un catalogue électronique, ça permet de garantir normalement que ça arrive dans le système du distributeur directement » (Ind N). Enfin, quand les distributeurs automatisent la réception et l'intégration des données dans leurs référentiels, celles-ci y sont 
intégrés plus rapidement que lors des ressaisies manuelles : ces ressaisies n'étaient pas toujours réalisées immédiatement à la réception des fiches produit dans le mode d'échange qui prédominait dans le secteur avant l'introduction des catalogues électroniques : "parfois, l'acheteur chez nous, il recevait une mise à jour, mais il était occupé à d'autres tâches plus urgentes, et du coup, la mise à jour pouvait être intégrée dans le référentiel après plusieurs jours d'attente. Voire jamais s'il l'oubliait! Là, l'acheteur reçoit une alerte automatique qui l'avertit et il n'a plus qu'à la valider pour qu'elle soit intégrée. S'il ne l'accepte pas, c'est qu'il y a un autre problème » (Dist $\mathrm{C}$ ). De même, quand les industriels automatisent l'émission, la fiche produit est envoyée plus rapidement au distributeur, ce qui permet d'améliorer la temporalité des données par la vitesse de transfert et d'intégration des données: «L'informatique va permettre d'accélérer considérablement les traitements, c'est clair » (Ind P). On retrouve dans le cas des fiches produit l'argument désormais classique d'un des avantages de l'EDI.

La centralisation des données est une contribution primordiale des différents catalogues électroniques qui interviennent pour l'échange des fiches produit: «La constitution d'un référentiel produit unique et complet est la première pierre que l'on peut poser pour travailler sur des catalogues » (Dist D). Quand les données sont centralisées, on s'assure de leur unicité lors de l'échange, et donc de leur objectivité : "Donc le but c'est de dire : un seul endroit où il y a l'information produit, et cette information produit elle est diffusable à tous les clients. Et on $s$ 'assure que l'information produit est correcte à cet endroit là. Parce qu'aujourd'hui, ce que font les directeurs de clientèle ou leur assistante, et bien ils renseignent le fichier comme ils l'entendent. Bien sûr ils font attention de ne pas envoyer n'importe quoi au client mais il n'empêche qu'ils ressaisissent les informations qu'ils ont, et ce n'est pas forcément les informations valides » (Ind $\mathrm{H})$; «l'avantage du PIM, c'est ce point d'entrée unique qui nous permet de distribuer ensuite l'information où il le faut » (Ind C). De plus, le stockage des données est centralisé à différentes étapes du processus d'échange, ce qui permet de les retrouver plus facilement, synonyme pour certaines entreprises d'amélioration de l'accessibilité des données : «ce que nous voulions, c'est que la base puisse être interrogée de partout » (Ind C). Enfin ce stockage centralisé est aussi pour certains un moyen d'améliorer la réputation de la source des données, notamment pour les industriels qui n'avaient pas de référentiel centralisé : «on connait la provenance des données [...] avant il fallait aller chercher telle donnée dans telle base, ce n'était pas structuré ! (Ind K). En effet, le récepteur ayant une meilleure visibilité de la provenance des données, il en est moins suspicieux vis-à-vis de leur qualité.

Parmi les fonctionnalités proposées par les catalogues électroniques, des contrôles automatiques sont présentés comme support d'amélioration de l'exactitude, de la crédibilité et de la complétude des données. Des contrôles de cohérence sont en effet menés pour détecter des données inexactes ou non crédibles aussi souvent que possible: «On a un outil [...] pour faire des contrôles automatiques de ratios. C'est vrai qu'on détecte beaucoup d'anomalies par ces contrôles de cohérence» (Dist E). De plus, l'utilisation de catalogues électroniques va de pair avec l'amélioration de la complétude des données, étant donné qu'une fiche produit avec un champ manquant sera désormais automatiquement refusée : «les contrôles automatiques [...] ça permet de vérifier qu'une donnée est présente ou pas présente (Dist G). Ainsi, "s'il y a un champ qui manque, ça ne va pas passer $\gg$ (Ind B)

L'implémentation de catalogues électroniques, du fait de leur utilisation pour automatiser les flux, est associée à la standardisation des fiches produit, que ce soit au niveau du modèle de données et au niveau de la signification des champs inclus dans le message. La standardisation des champs, aussi appelés attributs, permet d'améliorer la compréhensibilité des données. La standardisation des champs consiste à se mettre d'accord sur la signification des données échangées. Ainsi, si chaque 
participant conçoit un champ de la même façon que tous ses partenaires, il n'y a plus de risque de confusion ou de compréhension différente de l'un à l'autre. "Aujourd'hui, grâce au travail de GS1, quand on parle de largueur, hauteur et profondeur, on sait ce que c'est... La standardisation apporte beaucoup à ce niveau là » (Ind $\mathrm{E}$ ). La standardisation des fiches produit aussi est importante. Partager une structure de fiches produit, avec toujours les mêmes champs dans le même format, c'est une opportunité pour améliorer la consistance de la représentation des fiches produit : «avec la standardisation, on a le même format de données pour tous les distributeurs »(Ind D). Mais nous l'avons vu, certaines entreprises préfèrent utiliser un standard de fiches produit propriétaire, avec des champs hors standard pour améliorer la quantité des données qu'elles estiment pertinentes: "ce que ça apporte peut-être plus facilement, c'est de la richesse d'information. Typiquement, pour le livre, on a réussi de cette manière là à récupérer en automatique de manière systématique des informations complémentaires: l'auteur, la série, le titre» (Dist F) ; "VerticalWine, c'est une vraie plus value sur le vin, bien plus riche que le standard GS1 »(Dist G). Ainsi la standardisation de fiches produit au niveau sectoriel permet d'améliorer plus aisément la consistance de la représentation tandis que la standardisation de fiches produit propriétaires permet plutôt d'améliorer la quantité des données, leur valeur ajoutée et leur pertinence.

Enfin, l'introduction de TI pour structurer la synchronisation des données produit participe aussi au développement de la standardisation des processus d'échange. En effet, les échanges automatisés poussent les entreprises à définir conjointement les modalités d'échanges des fiches produit, depuis la définition des évènements déclencheurs de la transmission des données jusqu'à l'intégration des données dans les référentiels des distributeurs, en passant par la clarification des flux de données : «le flux est tracé et plus court. Ça évite de mettre en place des workflows complexes pour envoyer les informations produit à nos clients » (Ind $\mathrm{K}$ ) ; «il faut donc un alignement des données entre notre ERP et notre PIM, puis un alignement avec [notre SDP], puis un alignement avec le distributeur » (Ind $\mathrm{O}$ ). Dès lors, cette standardisation des processus participe à l'amélioration de l'exactitude, de la crédibilité, de la temporalité et de la complétude des données de la fiche produit. En effet, la mutualisation du transfert de la fiche produit vers plusieurs distributeurs permet à chacun d'entre eux et à l'industriel concerné de développer la qualité des données : "l'avantage aussi, c'est que si un de vos clients détecte une erreur, vous pouvez la corriger pour tous, automatiquement» (Ind A). La détection d'une erreur (donnée inexacte, invraisemblable ou manquante) par un des distributeurs ou par l'industriel autorise l'envoi d'une mise à jour de la fiche produit à tous les distributeurs avec des données d'une meilleure qualité, qui peut se juger en termes d'exactitude, de crédibilité, de complétude et de temporalité.

\section{Discussion}

Suite à la présentation des résultats, deux principales pistes de discussion sont ouvertes. Au-delà de la confirmation du caractère contextuel de la qualité des données et d'une définition des dimensions de la qualité des données dans le contexte particulier des fiches produit, l'autre piste est relative à la capacité des différentes formes de SIIO à soutenir l'amélioration de la qualité de données.

\subsection{Le caractère contextuel de la qualité des données}

Strong, Lee et Wang (1997) ont mis en évidence le caractère contextuel de la qualité des données en montrant que les dimensions qui la composent dépendent du contexte d'utilisation des données. Nous discutons dans cette partie des dimensions de la qualité des données dans le contexte 
spécifique des fiches produit, données à caractère contextuel (Legner et Schemm, 2008). C'est probablement du fait de la nature des données investiguées que nos résultats diffèrent des résultats classiques d'amélioration de la qualité des données par EDI. En effet, les recherches sur l'EDI ont surtout insisté sur la fiabilité des données et leur temporalité (voir par exemple Bergeron et Raymond, 1992 ; Chewlos et al., 2001 ; Baile et Hassairi, 2001 ; Li et Lin, 2006 ou Hartono et al., 2010). En effet, l'EDI supporte avant tout des échanges de messages à caractère transactionnel, pour lesquels les gains de productivité sur les processus associés sont issus de la vitesse d'exécution dans l'échange des données structurées fiables, faibles en volume mais avec une fréquence d'échange élevée. Concernant les fiches produit, dont le nombre de données est bien plus conséquent par message, dont la fréquence d'échange est bien moins élevée, dont les données sont très disparates puisque plus ou moins structurées, et dont l'échange a pour objectif de permettre une synchronisation des données entre les référentiels internes entre partenaires, on comprend aisément que le spectre des dimensions de la qualité des données concernées est de facto plus conséquent. En partant de la décomposition de Wang et Strong (1996), celle qui fait référence dans le monde académique (Madnick et al., 2009), et qui est suffisamment large et complète pour intégrer une grande variété de dimensions, nos résultats empiriques permettent de ré interroger les dimensions de la qualité des données qui apparaissent pertinentes aux yeux des acteurs dans le cas de la synchronisation des données. Parmi les 15 dimensions du modèle, certaines peuvent être regroupées et d'autres n'apparaissent pas dans le contexte de la fiche produit.

Certaines dimensions se regroupent sous la même notion. Ainsi, la crédibilité et l'exactitude se réfèrent à la notion de fiabilité des données. En effet, la qualité de certaines données est évaluée par leur exactitude, notamment les données d'identification et les données booléennes (présence d'un allergène, présence d'une matière dangereuse). D'autres données, comme les données logistiques des objets, admettent un certain degré de tolérance (GS1 et ECR, 2006). Enfin, certaines données alphanumériques, qui ne peuvent pas être exactes comme les libellés par exemple, seront évaluées sur leur crédibilité. Ainsi la fiabilité est le terme générique utilisé dans le secteur pour rendre compte de la capacité de chaque donnée à représenter correctement l'élément du monde réel auquel elle se réfère dans le système d'information (Wand et Wang, 1996). Nous pouvons aussi regrouper quantité, pertinence et valeur ajoutée. Dans l'étude empirique, puisque nous avons réalisé nos entretiens avec des personnes qui avaient sous leur responsabilité le projet d'électronisation des échanges, il nous est difficile de distinguer pertinence et valeur ajoutée : seuls les utilisateurs peuvent dire si une donnée n'est pas pertinente et nuit ainsi à la réalisation de la tâche ou si cette donnée apporte de la valeur pour réaliser la tâche plus rapidement et plus facilement. Par contre, dans le cas de la fiche produit, nous avons pu déterminer que le développement de la synchronisation des données ne permet pas d'améliorer pertinence et valeur ajouté pour des données qui étaient déjà transmises auparavant. C'est en ce sens qu'elles sont reliées à la dimension de quantité, à l'échange de données supplémentaires.

Dans le contexte de la fiche produit, deux dimensions identifiées par Wang et Strong (1996) ne se sont jamais présentées comme pouvant être améliorées. Il s'agit de l'interprétabilité et de la concision de la représentation. Pour l'interprétabilité, définie par des données qui se présentent dans la bonne langue et la bonne unité de mesure, la synchronisation des données n'apparaît pas comme une opportunité pour l'amélioration. Puisque nous nous sommes concentrés sur le marché français, la langue et l'unité de mesure des données ne sont pas modifiées par l'électronisation. Dans un entretien, nous avons pu voir que le problème pouvait se poser dans des pays multilingues, comme la Belgique. La concision de la représentation (les données sont présentées de façon suffisamment compacte pour éviter la surcharge) n'est pas présentée comme pouvant être améliorée. Cela vient notamment du fait que les fiches produit transférées dans une feuille Excel comportaient moins de champs : ainsi les données étaient représentées de façon plus concise, plus 
compacte. Par l'électronisation des échanges, la concision de la représentation est donc plutôt détériorée quand la quantité est améliorée. On trouve ici un exemple de conflit entre dimensions de la qualité des données. Ballou et Pazer (1995) avaient explicité le conflit entre exactitude et temporalité des données : on peut avoir des données exactes, mais à la temporalité non appropriée, et inversement. Ce conflit est ressorti de nos études de cas pour les données logistiques de la fiche produit : la transmission d'une mise à jour pour prévenir à l'avance d'une modification ne doit pas écraser les données précédentes avant la date du changement réel, sous peine de nuire à la fiabilité des données. D'autres conflits ponctuels (par exemple pertinence/temporalité, fiabilité/complétude, consistance/quantité) peuvent aussi émerger et nécessitent des règles de gestion adéquates pour garantir l'amélioration de la qualité des données dans sa globalité.

Enfin, la sécurité des données n'a été présentée qu'une seule fois comme pouvant être améliorée. Cette dimension est importante pour les acteurs et n'est pas occultée dans les discours, loin de là. La transmission des données privées, et notamment celles qui se réfèrent aux prix, nécessite une sécurité optimale des données. Elles ne doivent être accessibles que pour les deux entreprises concernées par cette relation. Mais pour la très grande majorité des entreprises, la migration vers un système de synchronisation n'est pas perçue comme une opportunité pour améliorer la sécurité des données. De plus, les avis sont partagés sur la capacité du SIIO à garantir la sécurité des données sensibles. Devant le caractère contextuel de la qualité des données, le tableau 3 présente une définition des dimensions de la qualité des données pour les fiches produit et pour lesquelles une amélioration est attendue.

Tableau 3 : Les dimensions de la qualité des données pour les fiches produit

\begin{tabular}{|l|l|}
\hline Dimension & Description (degré selon lequel :) \\
\hline Fiabilité & $\begin{array}{l}\text { les données de la fiche produit sont jugées correctes par rapport à ce qu'elles } \\
\text { représentent (exactes, avec une tolérance définie, ou vraisemblables selon le type de } \\
\text { données) }\end{array}$ \\
\hline Objectivité & les données de la fiche produit ne sont pas retraitées et sont uniques \\
\hline Réputation & la source des données est réputée fiable \\
\hline Complétude & les champs de la fiche produit sont remplis \\
\hline Temporalité & les données de la fiche produit sont certifiées à jour \\
\hline Valeur ajoutée & $\begin{array}{l}\text { le nombre de champs composant la fiche produit est approprié pour réaliser les tâches } \\
\text { auxquelles elles servent }\end{array}$ \\
\hline Compréhensibilité & $\begin{array}{l}\text { la définition des champs de la fiche produit est claire, et les données qui la composent } \\
\text { sont compréhensibles }\end{array}$ \\
\hline Consistance & les données de la fiche produit sont toujours représentées dans le même format \\
\hline Accessibilité & $\begin{array}{l}\text { les données de la fiche produit sont disponibles, facilement et rapidement trouvables } \\
\text { entre les référentiels des industriels et ceux des distributeurs }\end{array}$ \\
\hline
\end{tabular}

La suite de la discussion porte sur la conceptualisation des formes de SIIO émergentes, susceptibles de supporter l'amélioration de ces différentes dimensions de la qualité des données. 


\subsection{La capacité de diverses formes de SIIO à soutenir l'amélioration de la qualité des données}

Les résultats ont mis en évidence plusieurs configurations des SIIO pour réaliser la synchronisation des données. Dans l'interconnexion entre les industriels et les distributeurs utilisant un SDP et un RDP, les entreprises mettent chacune en place une base de données externe, qui peut être la même ou non. Il en résulte que la structure du SIIO de type A s'apparente à une structure de SIIO multilatéral où chaque entreprise met en place une liaison unique pour communiquer avec l'ensemble de ses partenaires (Choudhury, 1997), structure qui a été mise en évidence dans les recherches traitant des places de marché électronique (Soh et al., 2006 ; Dominguez, 2009). Quand on s'intéresse à l'interconnexion entre les industriels et les distributeurs n'utilisant pas de catalogues électroniques externes (RDP et SDP), le SIIO résultant s'apparente à un réseau de connexions dyadiques entre les PIM, bases de données internes des entreprises. C'est donc la dyade électronique (Choudhury, 1997) qui modélise la structure de type B, et qui a été longuement étudiée dans les recherches sur l'EDI (Iacovou et al., 1995 ; Baile et Hassairi, 2001 ; Subramani, 2004). Deux autres formes de SIIO (C et D) mettent en évidence une structure quelque peu différente des structures classiques présentées par Choudhury (1997) et par Kumar et van Dissel (1996). D'un point de vue conceptuel, on pourrait les rapprocher des formes de places de marché électroniques privées, ou en consortium restreint (Soh et al., 2006 ; Caby-Guillet et al., 2007 ; Dominguez, 2009), en mettant en évidence la superposition de l'intermédiation électronique privée par plusieurs concurrents vis-à-vis de leurs fournisseurs (ou clients) communs. Toutefois, cette structure renvoie à une interconnexion entre, d'un côté, des entreprises ayant choisi des liens dyadiques et de l'autre côté des entreprises ayant choisi des liens multilatéraux. C'est donc une structure de SIIO hybride qui émerge, structure hybride davantage mise en exergue dans la configuration de type $\mathrm{E}$ où des entreprises ayant la même position dans le réseau d'acteurs font des choix différents (de Corbière et Rowe, 2009). Ces structures hybrides peuvent être placées dans un continuum entre les deux extrêmes longuement analysés dans la littérature, comme le montre la figure 6 présentant une analyse configurationnelle en $2 * 2$ (Lyytinen et Damsgaard, 2011).

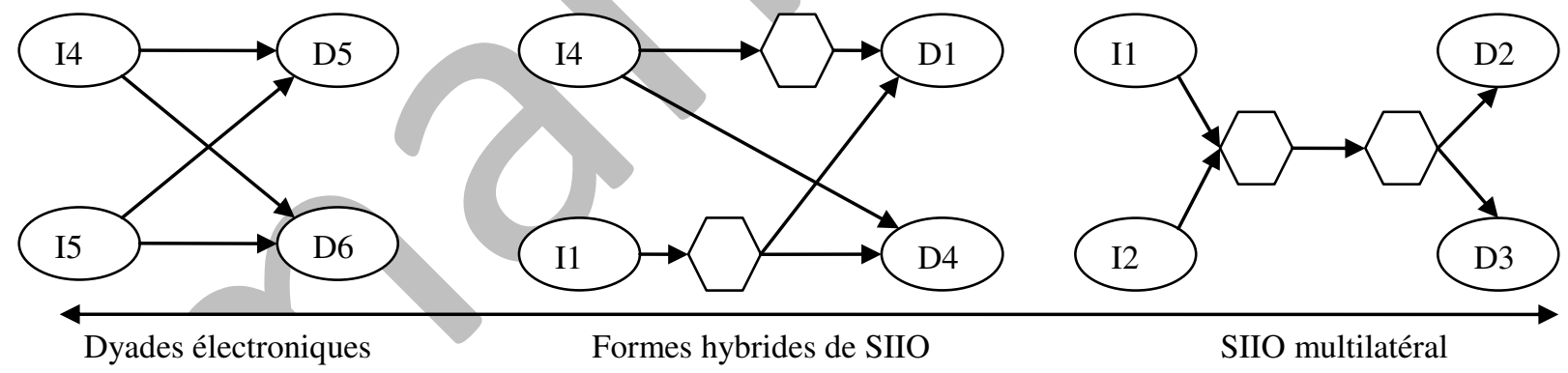

Figure 6 : Le continuum des SIIO (issu de Corbière et Rowe, 2009)

Dès lors il s'agit de comparer ces différentes formes de SIIO dans leurs capacités à soutenir l'amélioration de la qualité des données. De l'analyse sur les caractéristiques des SIIO permettant l'amélioration des différentes dimensions de la qualité des données évoquées précédemment, nous pouvons en conclure que les SIIO hybrides fournissent autant de garanties que les SIIO dyadiques et les SIIO multilatéraux. Quelle que soit sa position dans le continuum des SIIO, chaque forme de SIIO, reflétant un type d'interconnexion particulier entre les systèmes d'émission et de réception des données, possède les caractéristiques des SIIO permettant l'amélioration de la qualité des données. En effet, toutes les formes des SIIO du continuum peuvent être mises en œuvre avec la centralisation des données à différentes étapes du processus d'échange dans des technologies permettant la réalisation de contrôles informatiques. De plus, l'automatisation et la standardisation 
sont possibles et recommandées dans les flux de données entre ces différents outils dans les divers SIIO pour supporter la synchronisation de données. Par contre, certaines configurations par rapport à d'autres sont plus ou moins à même de supporter l'amélioration de certaines dimensions, reflétant des conflits entre elles (Ballou et Pazer, 1995). Ceci est particulièrement vrai pour les aspects de standardisation. En effet, le SIIO multilatéral favorise l'émergence de standards sectoriels quand le SIIO dyadique permet davantage de différenciation et donc l'adaptation des standards aux besoins de chaque participant, conduisant à des standards propriétaires (Christiansee et al, 2004 ; Markus et al., 2006; Zhu et al., 2006). Ainsi, les SIIO multilatéraux favorisent l'amélioration de la consistance de la représentation par le partage du modèle de données quand les SIIO dyadiques sont plus à même d'améliorer la valeur ajoutée des données inclues dans des messages propriétaires.

En synthèse, du tableau 2 et des explicitations de l'influence des caractéristiques des SIIO du continuum sur la qualité des données échangées, nous en tirons les propositions suivantes :

- l'automatisation des flux de données contribue à l'amélioration de la fiabilité, la temporalité et l'objectivité des données ;

- la centralisation des données dans les catalogues électroniques à différentes étapes du processus d'échange contribue à l'amélioration de l'objectivité, l'accessibilité et la réputation des données ;

- les contrôles informatiques dans les catalogues électroniques contribuent à l'amélioration de la fiabilité et la complétude des données ;

- la standardisation des données dans les messages échangés contribue à l'amélioration de la compréhensibilité, la consistance de la représentation et la valeur ajoutée des données ;

- la standardisation du processus d'échange contribue à l'amélioration de la fiabilité, la complétude et la temporalité des données.

\section{Conclusion}

Dans cet article, nous avons analysé la capacité des SIIO de synchronisation de données à supporter l'amélioration de la qualité des données. Ainsi, l'amélioration d'un certain nombre de dimensions de la qualité des données échangées a été associée à différentes caractéristiques des SIIO bâtis sur l'implémentation de catalogues électroniques: l'automatisation, les contrôles informatiques, la standardisation des données, la standardisation des processus et la centralisation des données à différentes étapes du processus d'échange. Au delà de cet apport, notre analyse permet d'une part de confirmer la multi dimensionnalité de la qualité des données (Wang et Strong, 1996) et d'autre part le caractère contextuel de la qualité des données (Strong et al., 1997). Elle ouvre la voie au développement de la connaissance académique sur la qualité des données dans le cadre des relations interorganisationnelles, sujet trop peu investi aujourd'hui (Nicolaou et McKnight, 2006). Il serait intéressant de réitérer l'analyse des effets de la mise en œuvre de SIIO sur l'amélioration de la qualité des données sous plusieurs aspects, par exemple en traitant des effets réels constatés par les entreprises, en diversifiant les processus analysés dans le même secteur, ou en comparant les résultats sur la synchronisation des données dans d'autres secteurs. De plus, grâce à l'analyse configurationnelle des SIIO (Lyytinen et Damsgaard, 2011) reflétant l'interconnexion entre des systèmes d'émission et de réception des données (de Corbière et Rowe, 2009), nous avons mis en exergue diverses formes de SIIO permettant la synchronisation des données, et notamment les formes hybrides que l'on peut placer dans un continuum entre deux formes extrêmes que sont les SIIO dyadiques et les SIIO multilatéraux (Choudhury, 1997). D'un point de vue théorique, il est particulièrement intéressant de noter que les diverses formes de SIIO du continuum sont toutes à même de supporter l'amélioration de la qualité des données. 
Concernant les limites de la recherche, nous nous sommes cantonnés à discuter des SIIO en fonction de la structure de l'échange issue des choix technologiques des entreprises en termes d'implémentation de catalogues électroniques. Ainsi focalisés sur l'interdépendance technique entre entreprises, nous n'avons pas distingué les typologies de Choudhury (1997) et de Kumar et van Dissel (1996). En ce sens, pour aller plus loin, en reprenant les travaux initiaux de Kumar et van Dissel (1996) développés par Clergeau et Rowe (2005), il serait intéressant de discuter des types de SIIO permettant l'échange de fiches produit en fonction du niveau d'interdépendance entre les entreprises. En effet, au delà du partage de la technologie, qu'en est-il en termes de partage de données et de processus? Retrouve-t-on des formes hybrides entre systèmes dyadiques et multilatéraux pour ces autres ressources potentiellement partagées par les entreprises ? Concrètement, quelle est l'influence de la nature des données dans l'organisation de leurs échanges? Comment s'organisent les flux de messages dans ces structures? De plus, le design de la recherche, élaboré pour identifier les améliorations attendues par les acteurs sur les dimensions de la qualité des données de Wang et Strong (1996) sans chercher à influencer les réponses, présente aussi certaines limites. Notamment, nous avons occulté les potentielles pertes de qualité, seulement abordées à travers les conflits entre dimensions.

D'un point de vue managérial, l'analyse empirique offre de nouvelles perspectives concernant la mise en place de SIIO. En effet, l'interconnexion effective entre des systèmes d'émission et de réception aux caractéristiques structurelles différentes met en évidence une plus grande flexibilité dans la construction des SIIO, et donc une plus grande valeur pour chaque entreprise dans le design du SIIO : la décision fondamentale pour les entreprises n'est plus relative à l'adoption d'un SIIO particulier, mais se réfère davantage à l'adoption d'un système ouvert vers ses partenaires (Truman, 2000), celui qui lui sied le mieux en fonction de ses contraintes. Le SIIO résultant issu de l'interconnexion avec les systèmes des partenaires émerge plus aisément grâce à la plus grande interconnectivité des technologies et standards modernes (Christiaanse et al., 2004 ; Zhu et al., 2006). De plus, cette recherche vient aussi compléter sous un angle théorique l'approche opérationnelle du Data Quality Challenge, groupe de travail mené par GS1 en collaboration avec L'Oréal, Sara Lee, Kamby, Auchan et Carrefour pour améliorer la qualité des données dans le secteur ${ }^{3}$. A travers l'identification des dimensions pouvant être impactées par les caractéristiques des SIIO, nous fournissons des arguments aux managers responsables de l'implémentation de TI pour justifier de la valeur de l'électronisation des échanges de données. Ainsi la mise en place de SIIO peut conduire à des bénéfices opérationnels directs sur la qualité des données échangées, qui eux-mêmes peuvent induire des bénéfices stratégiques sur l'efficience des relations interorganisationnelles de long terme (Yi et al., 2005). Enfin, la justification de la valeur des SIIO en termes d'amélioration de la qualité des données ne doit pas occulter que l'échange de données de qualité reste dépendant de la création de données de qualité et se doit de rester au service de l'utilisation de données de qualité.

\footnotetext{
${ }^{3}$ http://www.gs1.org/docs/gdsn/Data_Quality_Case_Study_France_final_April_2010.pdf
} 


\section{Bibliographie :}

Baile S., Hassairi F. (2002), "Impact de l'EDI sur la performance des équipementiers automobiles : l'apport de l'analyse de causalité", Système d'Information et Management, vol. 7, n 4, p. 5-42

Bailey J.P., Pearson S. (1983), "Development of a Tool for Measuring and Analysing Computer User Satisfaction", Management Science, vol. 29, n5, p. 530-545.

Ballou D., Pazer H. (1995), "Designing Information Systems to Optimize the Accuracy-Timeliness Tradeoff", Information System Research, vol. 6, n¹, p. 51-72.

Barrett S., Konsynski B. (1982), "Inter-organization Information Sharing Systems", MIS Quarterly, vol. 6, Special Issue, p. 93-105.

Bensaou M. (1997), "Interorganizational Cooperation: The Role of Information Technology - An Empirical Comparison of U.S. and Japanese Supplier Relations". Information Systems Research, vol. $8, \mathrm{n}^{\circ} 2$, p. 107-124.

Bergeron F., Raymond L. (1992), "The Advantages of Electronic Data Interchange". Data Base, vol. $23, \mathrm{n}^{\circ} 4$, p. 19-31.

Bernasconi M. (1996), "Les systèmes d'information inter-organisationnels sont-ils toujours source d'avantages concurrentiels durables ?", Systèmes d'Information et Management, vol. 1, nº1, p. 725 .

Caby-Guillet L., Clergeau C., de Corbière F., Dominguez C., Rowe F. (2007), "Perceived Value and Types of Electronic Marketplaces in the French Retail Industry: 5 Case Studies", The Communications of the Association for Information Systems, vol. 20.

Chewlos P., Benbasat I., Dexter A. (2001), "Research Report: Empirical Test of an EDI Adoption Model", Information Systems Research, vol. 12, n³, p. 304-321

Choudhury V. (1997), "Strategic Choices in the Development of Interorganizational Information Systems", Information Systems Research, vol. 8, $\mathrm{n}^{\circ}$ 1, p. 1-24.

Christiaanse E., Van Diepen T., Damsgaard J. (2004), "Proprietary versus Internet Technologies and the Adoption and Impact of Electronic Marketplaces", The Journal of Strategic Information Systems, vol. 13, p. 151-165.

Clergeau C., Rowe F. (2005), "Caractérisation des dispositifs d'interdépendance organisationnelle et mutualisation : le cas des centres d'appels virtuels", Systèmes d'Information et Management, vol. $10, \mathrm{n}^{\circ} 3$, p. 93-116.

de Corbière F., Rowe F. (2009), "Understanding the Diversity of IS Interconnections between Suppliers and Clients", JAIS Theory Development Workshop, December, Phoenix [AZ], USA.

de Corbière F., Rowe F. (2011), "Adoption Factors of Electronic Data Exchange and Technology: Can we distinguish Two Phases?", $19^{\text {th }}$ European Conference on Information Systems (ECIS), June, Helsinki, Finland.

Delone W.H., McLean E.R. (2003), "The DeLone and McLean Model of Information Systems Success: A Ten-Year Update", Journal of Management Information Systems, vol. 19, n4, p. 9-30.

Dominguez C. (2009), "Business models des places de marché électroniques : une taxonomie pour décrypter leurs enjeux", Systèmes d'Information et Management, vol. 14, n², p. 39-64

e-business (2005), Les cahiers du e-business, "centraliser l'information produit", n¹2, p.45-58. 
Elgarah W., Falaleeva N., Saunders C., Llie V., Shim J.T., Courtney J. (2005), "Data Exchange in Interorganizational Relationships: Review through Multiple Conceptual Lenses", Database for Advances in Information Systems, vol. 36, ${ }^{\circ} 1$, p. 8-29.

Filser M., des Garets V., Pache G. (2001), La distribution : organisation et stratégie, Editions Management et Société, Colombelles.

GS1 (2006), eCom Standards in the GS1 Community.

http://www.gs1.org/docs/ecom/eCom_Standards_in_the_GS1_Community_2006.pdf

GS1 et ECR (2006), Gestion des données synchronisée. Comment mettre en place de fiches produits standard et de qualité. Janvier 2006

Guilloux V. (1995), "Développement de l'EDI entre les fabricants et les distributeurs : impacts sur le schéma d'organisation du dialogue industrie-distribution", 2ème colloque de l'AIM (Association Information \& Management), Nanur, Belgique.

Hartono E., Li X., Na K-S, Simpson J.T. (2010), "The Role of the Quality of Shared Information in Interorganizational Systems Use", International Journal of Information Management, vol. 3, n5, 2010, p.399-407

Hlady-Rispal M. (2002), La méthode des cas, application à la recherche en gestion, De Boeck université, Bruxelles.

Iacovou C., Benbasat I., Dexter A. (1995), "Electronic Data Interchange and Small Organizations: Adoption and Impact of Technology". MIS Quarterly, vol.19, n 4, p.465-485.

Isaac H., Volle P. (2008), E-commerce - De la stratégie à la mise en oeuvre opérationnelle, Pearsons Education, Paris.

Kahn B.K., Pierce E.M., Melkas H. (2004), "IQ Research Directions", in Proceedings of the 2004 international conference on information quality, p.326-332.

Kumar K., Van Dissel H.G. (1996), "Sustainable Collaboration: Managing Conflict and Cooperation in Interorganizational Systems", MIS Quarterly, vol. 20, n³, p. 279-300.

Lee Y., Strong D.M., Kahn B., Wang R. (2002), "AIMQ: A Methodology for Information Quality Assessment", Information and Management, vol. 40, n², p. 133-146.

Legner C., Schemm J. (2008), "Toward the Inter-organizational Product Information Supply Chain - Evidence from the Retail and Consumer Goods Industries", Journal of the Association for Information Systems, Vol. 9, ${ }^{\circ} 4$, article 10.

Lepers X. (2003), Les relations d'échange entre la grande distribution et ses fournisseurs : le cas de l'enseigne Auchan, Thèse de doctorat, Sciences de gestion, Université Paris Dauphine.

Lesca H., Lesca E. (1995), Gestion de l'information : qualité de l'information et performances de l'entreprise, Litec, Paris.

Li S., Lin B. (2006), "Accessing Information Sharing and Information Quality in Supply Chain Management", Decision Support Systems, vol. 42, n³, p.1641-1656

Lyytinen K., Damsgaard J. (2011), "Inter-Organizational Information Systems Adoption - a Configuration Analysis Approach", European Journal of Information Systems, à paraître, http://www.palgrave-journals.com/ejis/journal/vaop/ncurrent/abs/ejis201071a.html

Madnick S., Wang R., Lee Y., Zhu H. (2009), "Overview and Framework for Data and Information Quality Research", ACM Journal of Data and Information Quality, vol. 1, n 1 , article 2. 
Malone T.W., Crowston K. (1990), "What is Coordination Theory and how can it Help Design Cooperative Work Systems ?", in Tatar D. (Ed.), Proceedings of the Third Conference on Computer-supported Cooperative Work, ACM Press, Los Angeles, CA, p. 357-370.

Malone T.W., Yates J., Benjamin R. (1987), "Electronic Markets and Electronic Hierarchies", Communications of the ACM, vol. 30, ${ }^{\circ}$ 6, p. 484-497.

Marciniak R., Rowe F. (2009), Systèmes d'information, dynamique et organisation (3e éd.), Economica, Paris.

Markus M.L., Steinfeld C., Wigand R., Minton G. (2006), "Industry-Wide Information Systems Standardization as Collective Action: the Case of the U.S. Residential Mortgage Industry", MIS Quarterly, vol. 30, special issue, p. 439-465.

Miles M.B., Huberman A.M. (2003), Analyse des données qualitatives, Trad. de la 2e éd. américaine, De Boeck, Paris.

Mukhopadhyay T., Kekre S. (2002), "Strategic and Operational Benefits of Electronic Integration in B2B Procurement Processes", Management Science, vol. 48, n¹0, p. 1301-1313.

Nakatani K., Chuang T.-T., Zhou D. (2006), "Data Synchronization Technology: Standards, Business Values and Implications", The Communications of the Association for Information Systems, vol. 17, p. 2-60.

Nicolaou A., McKnight H. (2006), "Perceived Information Quality in Data Exchanges: Effects on Risk, Trust, and Intention to use", Information Systems Research, vol. 17, n4, p. 332-351

Oliver C. (1990), "Determinants of Interorganizational Relationships: Integration and Future Directions", Academy of Management Review, vol. 15, n² 2, p. 241-265.

Porter M., Millar V.E. (1985), "How Information gives you Competitive Advantage?", Harvard Business Review, vol. 64, n²4, p. 149-160.

Rai A., Tang X. (2010), "Leveraging IT Capabilities and Competitive Process Capabilities for the Management of Interorganizational Relationship Portfolios", Information Systems Research, vol. $21, n^{\circ} 3$, p. 516-542.

Reix R. (2002), Systèmes d'information et management des organisations (4e éd.), Vuibert, Paris.

Robey D., Im G., Wareham J.D. (2008), "Theoretical Foundations of Empirical Research on Interorganizational Systems: Assessing Past Contributions and Guiding Future Directions", Journal of the Association for Information Systems, vol. 9, n 9 , p. 497-518.

Rodón J., Sesé F. (2010), "Analysing IOIS Adoption through Structural Contradictions", European Journal of Information Systems, vol. 19, nº, p. 637-648

Scannapieco M., Virgillito A., Marchetti C., Mecella M., Baldoni R. (2004), "The Architecture: a Platform for Exchanging and Improving Data Quality in Cooperative Information Systems", Information Systems, vol. 29, $\mathrm{n}^{\circ}$ 7, p. 551-582.

Soh C., Markus M.L., Huat G.K. (2006), "Electronic Marketplace and Price Transparency: Strategy, Information Technology and Success". MIS Quarterly, vol. 30, n³, p. 705-723.

Strong D.M., Lee Y.W., Wang R.Y. (1997), "Data Quality in Context", Communications of the $A C M$, vol. $40, \mathrm{n}^{\circ}$ 5, p. 103-110.

Subramani M. (2004), "How do Suppliers Benefit from Information Technology Use in Supply Chain Relationships?' MIS Quarterly, vol. 28, n 1 , p. 45-73. 
Truman G.E. (2000), "Integration in Electronic Exchange Environments", Journal of Management Information Systems, vol. 17, n¹, p. 209-244.

Van de Ven A.H., Walker G. (1984), "The Dynamics of Interorganizational Coordination", Administrative Science Quarterly, vol. 29, n4, p. 598-621.

Wand Y., Wang R.Y. (1996), "Anchoring Data Quality in Ontological Foundations", Communications of the ACM, vol. 9, $\mathrm{n}^{\circ} 11$, p. 86-95.

Wang R.Y., Strong D. (1996), "Beyond Accuracy: What Data Quality Means to Data Consumers", Journal of Management Information Systems, vol. 12, n 4, p. 5-34.

Williamson O.E. (1985), The Economic Institutions of Capitalism: Firms, Markets, Relational Contracting, The Free Press, New-York

Yi Y., Soh C., Huang L. (2005) "Strategic and Operational Benefits of IOS-Enabled Interorganizational Integration", the $26^{\text {th }}$ International Conference on Information Systems (ICIS), December, Las Vegas, USA.

Yin R.K. (2003), Case Study Research: Design and Methods (3rd ed), Sage, Thousand Oaks.

Zhu K., Kraemer K.L., Gurbaxani V., Xu S. (2006), "Migration to Open-Standard Interorganizational Systems: Network Effects, Switching Costs and Path Dependency", MIS Quarterly, vol. 30, Special issue, p. 515-539.

Zmud R. (2005), "Préface" in Wang R., Pierce E., Madnick S., Fisher, C. (Eds.), Information Quality (Advances in Management Information Systems), M.E. Sharpe, Armonk, NY. 
Annexe 1 : Description des dimensions de la qualité des données (Wang et Strong, 1996)

\begin{tabular}{|l|l|}
\hline Dimension & Description. Degré selon lequel les données : \\
\hline Exactitude & sont correctes, certifiées sans erreurs par rapport à ce qu'elles représentent \\
\hline Crédibilité & sont perçues comme étant vraisemblables et crédibles \\
\hline Objectivité & sont considérées comme non biaisées et impartiales \\
\hline Réputation & ont une source réputée fiable \\
\hline Valeur ajoutée & apportent des avantages par leur utilisation \\
\hline Pertinence & permettent d'aider à la réalisation de la tâche \\
\hline Complétude & sont suffisamment profondes et larges pour la tâche \\
\hline Temporalité & ont un âge approprié pour la tâche \\
\hline Quantité & sont suffisamment nombreuses pour réaliser la tâche \\
\hline Interprétabilité & se présentent dans la bonne langue et la bonne unité de mesure \\
\hline Compréhensibilité & sont claires, sans ambiguïté et facilement compréhensibles \\
\hline Consistance & sont toujours présentées dans le même format \\
\hline Concision & sont présentées de façon suffisamment compacte pour éviter la surcharge \\
\hline Accessibilité & sont disponibles, facilement et rapidement trouvables \\
\hline Sécurité & ont un accès restreint de façon approprié \\
\hline
\end{tabular}


$>$ Présentation de l'interlocuteur et de son entreprise

$>$ Stratégie et objectifs de l'entreprise

- Question d'ouverture: Pouvez vous me décrire l'historique des échanges de fiches produit de votre entreprise?

- Thèmes à aborder :

- les méthodes traditionnelles : Comment échangiez-vous les fiches produit avant l'apparition des outils comme les catalogues électroniques et les PIM ?

- les raisons qui poussent à l'adoption ou à la non adoption d'un SIIO : pourquoi avez-vous décidé de vous orienter vers des échanges électroniques ? ou pourquoi conserver des méthodes d'échanges traditionnels?

- sur le type de produits : Pour vous, l'échange est conçu pour les

SIIO produits permanents ou pour les promotions?

- Question d'ouverture: Quels sont les technologies que vous avez choisies?

- Thèmes à aborder:

- sur les raisons du choix (architectures, outils, standards)

- Pourquoi tel choix plutôt qu'un autre ?

- Qu'est ce qui a influencé ce choix ?

- sur les processus associés

- Qu'est ce qui change par rapport aux transmissions traditionnelles (réorganisation interne et externe) ?

- Quels acteurs interviennent dans l'échange ?

- Quel événement déclenche l'envoi ou la réception de la fiche?

- sur le standard: Qu'en pensez vous ? Correspond t'il à vos besoins? Qu'est ce qu'il lui manque?

- sur le retour d'expérience : Quel est le retour d'expérience sur les outils et technologies? Quels points positifs ? Quelles difficultés rencontrées ? A quel niveau ? Pourquoi ? Comment les résoudre?

\section{La qualité des données}

- Question d'ouverture: Quels sont les types de problèmes que l'entreprise rencontre à cause d'une mauvaise qualité de données ?

- Questions de relance : est ce que vous pouvez me donner des exemples ? Vous m'avez parlé tout à l'heure de tel problème...

- Thème sur le SIIO : qu'attendez-vous des outils mis en place par rapport à ces différents problèmes

- Thème sur la réalisation: sous quelles conditions pensez-vous réussir à améliorer la qualité des données ?

\section{Conclusion}

○ Finalement pour vous, comment définir une donnée ou une fiche produit de qualité ? 


\section{Annexe 3 : Description des entretiens}

Le tableau ci-dessous indique le nombre d'entretiens réalisés dans chacune des entreprises analysées.

\begin{tabular}{|c|c|c|c|c|c|}
\hline Dist A & 1 & Ind A & 1 & Ind J & 2 \\
\hline Dist B & 3 & Ind B & 2 & Ind K & 1 \\
\hline Dist C & 4 & Ind C & 1 & Ind L & 1 \\
\hline Dist D & 2 & Ind D & 1 & Ind M & 3 \\
\hline Dist E & 2 & Ind E & 1 & Ind N & 1 \\
\hline Dist F & 1 & Ind F & 2 & Ind O & 1 \\
\hline Dist G & 1 & Ind G & 1 & Ind P & 2 \\
\hline & & Ind H & 2 & Ind Q & 1 \\
\hline & & Ind I & 1 & Ind R & 2 \\
\hline
\end{tabular}

Concernant le profil des personnes interviewées, comme précisé dans la partie méthodologie, toutes ont sous leur responsabilité tout ou partie des projets d'électronisation des échanges de fiches produit, d'implémentation de catalogues électroniques.

Seuls 5 des 40 interviewés sont rattachés aux directions métiers, telles que Supply Chain, Achats, Relation Clients, avec des fonctions relatives à la gestion des informations dans ces directions. Ainsi, la très grande majorité des interviewés provient des directions IT, SI ou informatique. Parmi ces 35 interviewés, des profils très différents peuvent être identifiés, en termes de fonction/poste (Directeur, Chef de projet, Chargé de mission, Responsable, Manager) et en termes de périmètre (DSI, échanges BtoB, études informatiques, catalogue électronique, PIM, GDS, EDI, référentiel, fiche produit, synchronisation des données, standards, Master Data Manager). 